Pure and Applied Mathematics Quarterly

Volume 2, Number 2

(Special Issue: In honor of

John H. Coates, Part 2 of 2$)$

465-496, 2006

\title{
Iwasawa Descent and Co-descent for Units modulo Circular Units
}

\author{
T. Nguyen Quang Do and M. Lescop \\ (with an appendix by J.-R. Belliard) \\ To John Coates for his $60^{\text {th }}$ birthday.
}

\begin{abstract}
Let $F$ be a totally real abelian number field, $F_{\infty}=\cup_{n \geqslant 0} F_{n}$ its cyclotomic $\mathbb{Z}_{p}$-extension $(p \neq 2), \Gamma_{n}=\operatorname{Gal}\left(F_{\infty} / F_{n}\right)$. Let $B_{n}$ be the $p$ part of the quotient $U_{n} / C_{n}$ of the units of $F_{n}$ modulo circular units. We study descent and co-descent for the Iwasawa modules $B_{\infty}=\lim B_{n}$ and $Y_{\infty}=\lim B_{n}$; more precisely, using the Main Conjecture, we determine the kernels and cokernels of the natural maps $B_{n} \rightarrow\left(B_{\infty}\right)^{\Gamma_{n}}$ and $\left(Y_{\infty}\right)_{\Gamma_{n}} \rightarrow B_{n}$ in terms of some (module theoretic) invariants attached to $F_{\infty} / F$. We derive some consequences related to Greenberg's conjecture.
\end{abstract}

\section{INTRODUCTION}

Let $F$ be any number field and $p$ an odd rational prime. Denote by $F_{\infty}$ the cyclotomic $\mathbb{Z}_{p}$-extension of $F$, with finite layers $F_{n}$, and by $\Gamma_{n}$ (respectively $\Gamma$ ) the Galois group of $F_{\infty} / F_{n}$ (resp. $F_{\infty} / F$ ). Let $A_{n}$ be the $p$-Sylow subgroup of the ideal class group of $F_{n}$ and consider the usual Iwasawa modules $A_{\infty}:=\lim A_{n}$ (w.r.t. extension maps) and $X_{\infty}:=\lim A_{n}$ (w.r.t. norm maps). A classical (and mainly settled) topic in Iwasawa theory consists in studying descent and codescent for the modules $A_{\infty}$ and $X_{\infty}$, more precisely in determining the kernels and cokernels of the natural maps $A_{n} \rightarrow\left(A_{\infty}\right)^{\Gamma_{n}}$ and $\left(X_{\infty}\right)_{\Gamma_{n}} \rightarrow A_{n}$ (see e.g. [Iw2], [Ku], [LMN],...). Suppose now that $F$ is totally real abelian. Then, for any $n \geqslant 0$, the group of units $U_{n}$ of $F_{n}$ contains a remarkable subgroup $C_{n}$ consisting of the so-called circular units of $F_{n}$ in the sense of Sinnott ([S]). Let $B_{n}$ be the $p$-part of the "unit class group" $U_{n} / C_{n}, B_{\infty}=\underline{\lim } B_{n}$ (the transition maps

Received October 22, 2005.

Classification: 11R23; key words : circular units, unit class group. 
being induced by inclusion), $Y_{\infty}=\varliminf^{\lim } B_{n}$ (the transition maps being induced by norms). A strong parenthood exists between the ideal class groups and the unit class groups, as is suggested by Sinnott's index formula, which implies that the orders $\left|A_{n}\right|$ and $\left|B_{n}\right|$ are asymptotically equivalent, or by the Main Conjecture (i.e. Mazur-Wiles' theorem), which implies that the $\Lambda$-modules $X_{\infty}$ and $Y_{\infty}$ have "the" same characteristic series. In spite of this, the problem of descent and codescent has received much less attention in the unit class setting than in the ideal class setting. To the best of our knowledge:

- for co-descent, the kernels and cokernels of the natural projection maps $h_{n}$ : $\left(Y_{\infty}\right)_{\Gamma_{n}} \rightarrow B_{n}$ have been shown to be bounded (uniformly w.r.t. $n$ ) in $[\mathrm{N}]$ using the Main Conjecture and in [B3] using only Leopoldt's conjecture; see also the appendix in [FG]. But except in particular cases (as in [O.I], [O.II]), a precise description of these kernels and cokernels is missing.

- for descent, the kernels of the natural extension maps $j_{n}: B_{n} \rightarrow\left(B_{\infty}\right)^{\Gamma_{n}}$ are known ([KN], [BN2]), but the cokernels have not been touched upon.

An extra difficulty in the unit class setting arises from the fact that we miss the Galois theoretic interpretation of the ideal class groups given by class field theory. It is known to experts that a precise solution should come from an accurate enough description of the cohomology of units and circular units along the cyclotomic tower, and this is exactly what we intend to do in this article, modulo a (mildly) restrictive hypothesis called (DG) (see 2.1 below) on Galois descent for the circular units themselves. An appendix by J.-R. Belliard gives a criterion for condition (DG) to hold - which happens in most "usual" cases. In order not to overload the results with useless technical generalities, we shall also assume throughout a (not really) restrictive hypothesis on ramification

(R) The $p$-primes of $F$ are totally ramified in $F_{\infty}$.

Then the number $s$ of primes above $p$ is the same for $F$ and $F_{\infty}$.

The solutions to the descent and co-descent problems will be given in terms of some asymptotic (module theoretic) invariants attached to $F_{\infty} / F$ (see theorems 3.7 and 4.5 ). In spite of the simplifying hypothesis (R), some general statements remain rather cumbersome or partially inconclusive, which explains why we shall illustrate them systematically by the detailed corresponding results (which will also present the advantage of being non asymptotic) obtained in the following particular case: 
(A) $F$ admits only one $p$-prime, which is totally ramified in $F_{\infty} / F$.

Note that $(\mathrm{A})$ is equivalent to $(\mathrm{R})$ and $s=1$. The archetypical examples for (A) are of course the subfields of $\mathbb{Q}\left(\zeta_{p}+\zeta_{p}^{-1}\right)$.

Here is a list of notations which will be used throughout:

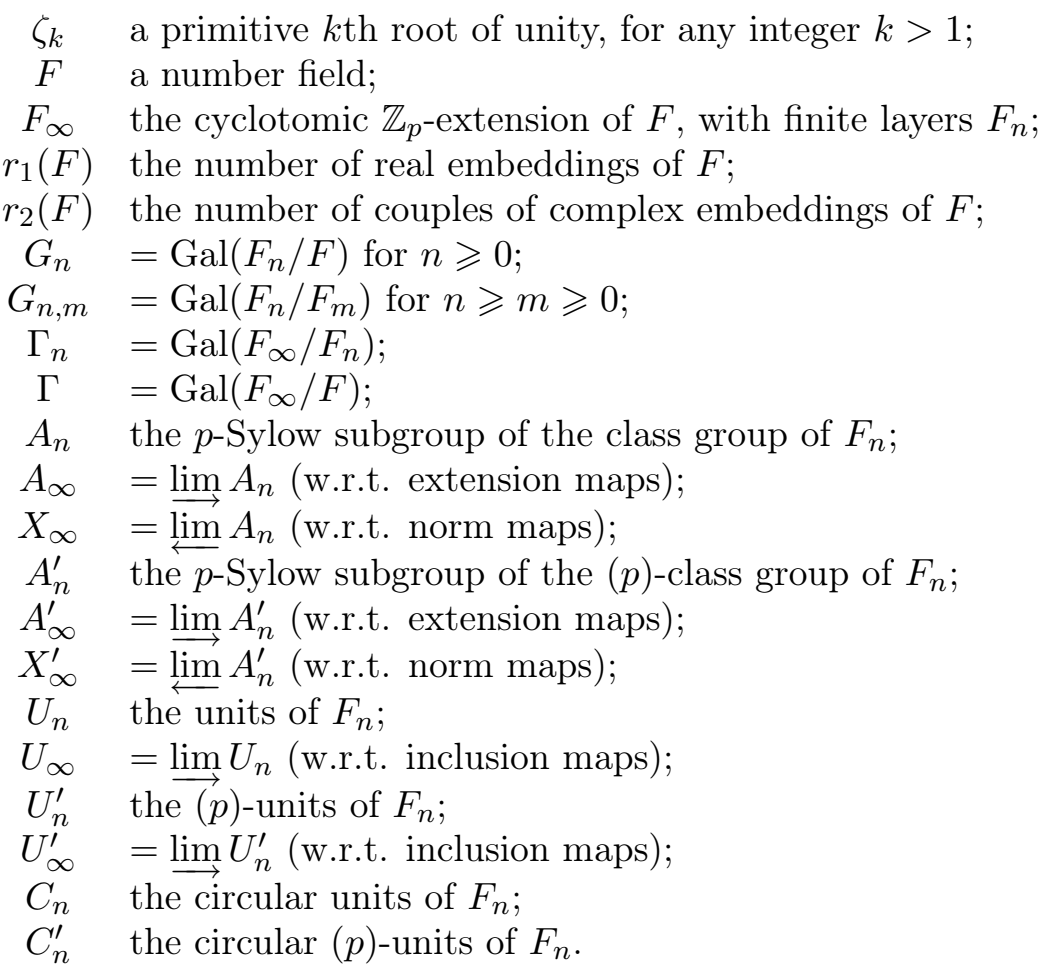

Moreover, for any abelian group $A$, we shall denote by $\bar{A}=\underset{q}{\lim } A / A^{p^{q}}$ the $p$ completion of $A$. If $A$ is finitely generated, then $\bar{A}=A \otimes \mathbb{Z}_{p}$. Thus let us define

$$
\begin{aligned}
\bar{U}_{\infty} & =\lim _{\bar{U}_{n}} \text { (w.r.t. norms); } \\
\bar{U}_{\infty}^{\prime} & =\lim _{\longleftarrow} \bar{U}_{n}^{\prime} \text { (w.r.t. norms). }
\end{aligned}
$$

Finally, for any group $G$ and any $G$-module $M$, let $M^{G}$ be the $G$-invariants of $M$, namely the points of $M$ fixed by the action of $G$, and $M_{G}$ the $G$-coinvariants of $M$, namely the largest quotient of $M$ on which $G$ acts trivially. 


\section{Capitulation kernels and Cohomology of units}

In this section, $F$ is any number field such that all the layers $F_{n}$ satisfy Leopoldt's and Gross' conjectures at $p$. We aim to compute the $G_{n}$-cohomology of $U_{n}$ and $U_{n}^{\prime}$ or, by default, the $\Gamma$-cohomology of $U_{\infty}$ and $U_{\infty}^{\prime}$. We may remark right from the start that, because $G_{n}$ is a $p$-group and $U_{n}$ and $U_{n}^{\prime}$ are finitely generated over $\mathbb{Z}$, we have $\hat{H}^{i}\left(G_{n}, U_{n}\right) \simeq \hat{H}^{i}\left(G_{n}, \bar{U}_{n}\right)$ for all $i \in \mathbb{Z}$, and similarly $\hat{H}^{i}\left(G_{n}, U_{n}^{\prime}\right) \simeq \hat{H}^{i}\left(G_{n}, \bar{U}_{n}^{\prime}\right)$.

Before proceeding any further, let us recall three important results related to inverse limits of units and $(p)$-units and the Gross conjecture:

(a) Sinnott's exact sequence:

For any prime $v$ of $F$ dividing $p$, let $F_{v}$ be the completion of $F$ at $v$ and let $\hat{F}_{v}^{\times}$be the group of universal norms of the cyclotomic $\mathbb{Z}_{p}$-extension $F_{v, \infty}$ of $F_{v}$. Precisely, $\hat{F}_{v}^{\times}$is the subgroup of $\overline{F_{v}^{\times}}$which corresponds by class field theory to $F_{v, \infty}$; in other words, $\overline{F_{v}^{\times}} / \hat{F}_{v}^{\times} \simeq \operatorname{Gal}\left(F_{v, \infty} / F_{v}\right)$. Sinnott's exact sequence ([FGS], $[\mathrm{Ko}], \ldots$ ) reads:

$$
0 \rightarrow \hat{U}_{F}^{\prime} \rightarrow \bar{U}_{F}^{\prime} \stackrel{\operatorname{diag}}{\longrightarrow} \underset{v \mid p}{\tilde{\oplus}} \overline{F_{v}^{\times}} / \hat{F}_{v}^{\times} \stackrel{\operatorname{Artin}}{\longrightarrow}\left(X_{\infty}^{\prime}\right)_{\Gamma} \rightarrow A_{F}^{\prime}
$$

Here, $\tilde{\oplus}$ denotes the kernel of the map "sum of components" and the "Gross kernel" $\hat{U}_{F}^{\prime}$ is defined as the kernel of the map diag above. All other notations are obvious. The Gross kernel actually consists of all elements of $\bar{U}_{F}^{\prime}$ which are everywhere local universal norms (w.r.t. the cyclotomic $\mathbb{Z}_{p}$-extension of $F$ ). Recall ([FGS], $[\mathrm{J}],[\mathrm{Ko}], \ldots)$ that the Gross conjecture for $F$ at $p$ states that $\left(X_{\infty}^{\prime}\right)_{\Gamma}$ (or equivalently $\left.\left(X_{\infty}^{\prime}\right)^{\Gamma}\right)$ is finite. It is known to hold if $F$ is abelian.

(b) The $\Lambda$-freeness of some inverse limits:

Recall that $\bar{U}_{\infty}^{\prime}:=\lim _{U_{n}}^{\prime}$ (w.r.t. norms). It is obvious that its torsion submodule, tor $\bar{U}_{\infty}^{\prime}$, is $\mathbb{Z}_{p}(1)$ or trivial according as $F$ contains or not a primitive $p$ th root of unity. Let $\operatorname{Fr}_{\Lambda} \bar{U}_{\infty}^{\prime}:=\bar{U}_{\infty}^{\prime} / \operatorname{tor}_{\Lambda} \bar{U}_{\infty}^{\prime}$. Then Kuz'min has shown ([Ku], proposition 7.5) that $\operatorname{Fr}_{\Lambda} \bar{U}_{\infty}^{\prime}$ is $\Lambda$-free of rank $r_{1}(F)+r_{2}(F)$. By purely algebraic arguments, it follows that $\operatorname{Fr}_{\Lambda} \bar{U}_{\infty}$ is also $\Lambda$-free of the same rank ([BN2], 2.1).

(c) The obstruction to Hasse's norm principle: 
Kuz'min has shown $([\mathrm{Ku}]$, theorem 7.2$)$ that $\tilde{U}_{F}^{\prime}:=\left(\operatorname{Fr}_{\Lambda} \bar{U}_{\infty}^{\prime}\right)_{\Gamma}$ injects into $\hat{U}_{F}^{\prime}$, with cokernel isomorphic to $\left(X_{\infty}^{\prime}\right)^{\Gamma}$. Let $X_{\infty}^{\prime}$ tor denote the maximal finite submodule of $X_{\infty}^{\prime}$. Modulo Gross' conjecture, Kuz'min's theorem reads:

$$
\hat{U}_{F}^{\prime} / \tilde{U}_{F}^{\prime} \simeq\left(X_{\infty}^{\prime t o r}\right)^{\Gamma}
$$

We have seen that the Gross kernel $\hat{U}_{F}^{\prime}$ consists of everywhere local universal norms, and the $\Lambda$-freeness of $\operatorname{Fr}_{\Lambda} \bar{U}_{\infty}^{\prime}$ implies that $\tilde{U}_{F}^{\prime}$ consists of global universal norms. Thus $\left(X_{\infty}^{\prime}{ }^{t o r}\right)^{\Gamma}$ can be considered as an obstruction to Hasse's norm principle for the cyclotomic $\mathbb{Z}_{p}$-extension $F_{\infty} / F$.

We are now ready to compute the cohomology of the units and $(p)$-units along the cyclotomic tower. The easier case is that of the $(p)$-units because, since $F_{\infty} / F$ is unramified outside $p$, we are actually in an étale setting.

\section{Theorem 1.1.}

(i) For all $n \geqslant 0$, we have a capitulation exact sequence:

$$
0 \rightarrow H^{1}\left(G_{n}, U_{n}^{\prime}\right) \rightarrow A_{0}^{\prime} \rightarrow\left(A_{n}^{\prime}\right)^{G_{n}} \rightarrow H^{2}\left(G_{n}, U_{n}^{\prime}\right) \rightarrow H^{2}\left(G_{n}, F_{n}^{\times}\right) .
$$

(ii) $H^{1}\left(\Gamma, U_{\infty}^{\prime}\right) \simeq \operatorname{Ker}\left(A_{0}^{\prime} \rightarrow\left(A_{\infty}^{\prime}\right)^{\Gamma}\right)$ and $H^{2}\left(\Gamma, U_{\infty}^{\prime}\right) \simeq\left(\mathbb{Q}_{p} / \mathbb{Z}_{p}\right)^{s-1}$. Recall that $s$ denotes the number of p-primes of $F$ or $F_{\infty}$.

(iii) The modules $H^{1}\left(\Gamma_{n}, U_{\infty}^{\prime}\right)$ stabilize for $n$ large enough. More precisely, via (ii) and norm maps, they become isomorphic to $X_{\infty}^{\prime}$ tor , the maximal finite submodule of $X_{\infty}^{\prime}$.

Proof. The statements (i) to (iii) are known results from [Iw1], [Iw2], [Ku], [LMN] etc... We'll reprove only the isomorphism $H^{2}\left(\Gamma, U_{\infty}^{\prime}\right) \simeq\left(\mathbb{Q}_{p} / \mathbb{Z}_{p}\right)^{s-1}$, following an approach which will be used again in the sequel. Let us first determine the $G_{n^{-}}$ cohomology of the $\hat{U}_{n}^{\prime}$ 's

Lemma 1.2. For all $n \geqslant 0$ and for all $i \in \mathbb{Z}$, we have canonical isomorphisms:

$$
\hat{H}^{i-2}\left(G_{n},\left(X_{\infty}^{\prime}\right)_{\Gamma_{n}}\right) \simeq \hat{H}^{i}\left(G_{n}, \hat{U}_{n}^{\prime}\right) \simeq \hat{H}^{i}\left(G_{n},\left(X_{\infty}^{\prime}{ }^{\text {tor }}\right)^{\Gamma_{n}}\right) .
$$

Proof. By Kuz'min's result (b), $\tilde{U}_{n}^{\prime}$ is $G_{n}$-cohomologically trivial hence, by (c), $\hat{H}^{i}\left(G_{n}, \hat{U}_{n}^{\prime}\right) \simeq \hat{H}^{i}\left(G_{n},\left(X_{\infty}^{\prime}{ }^{\text {tor }}\right)^{\Gamma_{n}}\right)$. Let us show now that $\hat{H}^{i-2}\left(G_{n},\left(X_{\infty}^{\prime}\right)_{\Gamma_{n}}\right) \simeq$ $\hat{H}^{i}\left(G_{n},\left(X_{\infty}^{\prime}{ }^{\text {tor }}\right)^{\Gamma_{n}}\right)$. Let $\mathfrak{X}_{\infty}$ be the Galois group, over $F_{\infty}$, of the maximal $p$ ramified abelian pro- $p$-extension of $F_{\infty}$ and let us define $\mathfrak{X}_{\infty, v}:=\lim _{\longleftarrow} \overline{F_{n, v_{n}}^{\times}}$, where $v$ is any $p$-adic prime of $F$ and $v_{n}$ is the unique prime of $F_{n}$ above $v$. By class field theory, we have an exact sequence: 


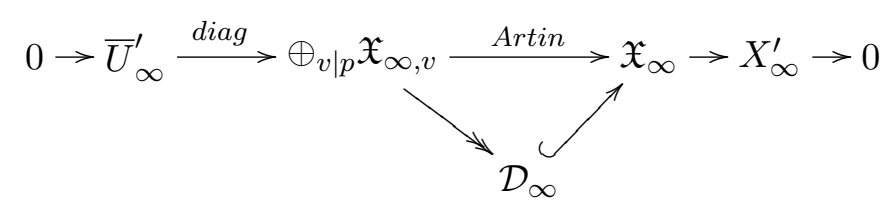

where $\mathcal{D}_{\infty}$ is by definition the cokernel of the map diag. Note that the map diag is injective because of the validity of Leopoldt's weak conjecture. Recall that one of the many equivalent versions of Leopoldt's conjecture for $F_{n}$ states that $\mathfrak{X}_{\infty}^{\Gamma_{n}}=(0)$, so we get by the snake lemma:

$$
0 \rightarrow\left(X_{\infty}^{\prime}\right)^{\Gamma_{n}} \rightarrow\left(\mathcal{D}_{\infty}\right)_{\Gamma_{n}} \rightarrow\left(\mathfrak{X}_{\infty}\right)_{\Gamma_{n}} \rightarrow\left(X_{\infty}^{\prime}\right)_{\Gamma_{n}} \rightarrow 0 .
$$

But $\mathfrak{X}_{\infty}$ has no non-trivial finite submodule, so that the projective dimension $\operatorname{pd}_{\Lambda}\left(\mathfrak{X}_{\infty}\right)$ over $\Lambda$ of $\mathfrak{X}_{\infty}$ is less than or equal to 1 , i.e. we have an exact sequence of the form

$$
0 \rightarrow \Lambda^{r} \rightarrow \Lambda^{d} \rightarrow \mathfrak{X}_{\infty} \rightarrow 0,
$$

with positive integers $r$ and $d$. The snake lemma then gives

$$
\mathfrak{X}_{\infty}^{\Gamma_{n}}=(0) \rightarrow \mathbb{Z}_{p}\left[G_{n}\right]^{r} \rightarrow \mathbb{Z}_{p}\left[G_{n}\right]^{d} \rightarrow\left(\mathfrak{X}_{\infty}\right)_{\Gamma_{n}} \rightarrow 0,
$$

hence the cohomological $G_{n}$-triviality of $\left(\mathfrak{X}_{\infty}\right)_{\Gamma_{n}}$. But $\mathcal{D}_{\infty} \subset \mathfrak{X}_{\infty}$, and so the same works for $\left(\mathcal{D}_{\infty}\right)_{\Gamma_{n}}$, hence the isomorphism $\hat{H}^{i-2}\left(G_{n},\left(X_{\infty}^{\prime}\right)_{\Gamma_{n}}\right) \simeq \hat{H}^{i}\left(G_{n},\left(X_{\infty}^{\prime}\right)^{\Gamma_{n}}\right)$.

Resuming the proof of 1.1 (iv), let us consider the quotient $V_{n}:=\bar{U}_{n}^{\prime} / \hat{U}_{n}^{\prime} \hookrightarrow$ $\underset{v \mid p}{\tilde{\oplus}} \overline{F_{n, v}^{\times}} / \hat{F}_{n, v}^{\times}$. By hypothesis (R), $G_{n}$ acts trivially on the set of $p$-primes of $F_{n}$, and

for such a $p$-prime $v$, the decomposition group $G_{n, v}$ acts trivially on $\operatorname{Gal}\left(F_{\infty, v} / F_{v}\right)$, hence in particular $G_{n}$ acts trivially on $V_{n} \simeq \mathbb{Z}_{p}^{s-1}$. Then the tautological exact sequence $0 \rightarrow \hat{U}_{n}^{\prime} \rightarrow \bar{U}_{n}^{\prime} \rightarrow V_{n} \rightarrow 0$ gives rise to an exact sequence

$$
H^{2}\left(G_{n}, \hat{U}_{n}^{\prime}\right) \rightarrow H^{2}\left(G_{n}, \bar{U}_{n}^{\prime}\right) \rightarrow H^{2}\left(G_{n}, V_{n}\right) \simeq\left(\mathbb{Z} / p^{n} \mathbb{Z}\right)^{s-1} \rightarrow H^{3}\left(G_{n}, \hat{U}_{n}^{\prime}\right)
$$

Applying lemma 1.2 and taking inductive limits on $n$, we get an exact sequence:

$$
H^{2}\left(\Gamma, X_{\infty}^{\prime}{ }^{\text {tor }}\right) \rightarrow H^{2}\left(\Gamma, U_{\infty}^{\prime}\right) \rightarrow\left(\mathbb{Q}_{p} / \mathbb{Z}_{p}\right)^{s-1} \rightarrow H^{3}\left(\Gamma, X_{\infty}^{\prime}{ }^{\text {tor }}\right) .
$$

The two extreme terms are null because $\operatorname{cd}_{p}(\Gamma)=1$.

The cohomology of the units is not so simple to handle. It is known for $F$ totally real $\left([\mathrm{Iw} 2]\right.$, p.197) that $H^{2}\left(\Gamma, U_{\infty}\right) \simeq\left(\mathbb{Q}_{p} / \mathbb{Z}_{p}\right)^{s-1}$ and $H^{1}\left(\Gamma, U_{\infty}\right) \sim\left(\mathbb{Q}_{p} / \mathbb{Z}_{p}\right)^{s}$ but, for our purposes, we need to make the latter pseudo-isomorphism more precise. The main parameters in our calculation will be the kernels $D_{n}:=\operatorname{Ker}\left(A_{n} \rightarrow A_{n}^{\prime}\right)$; in other words, $D_{n}$ is the image in $A_{n}$ of the subgroup of ideals generated by the primes of $F_{n}$ above $p$. By hypothesis (R), $G_{n}$ acts trivially on $D_{n}$. If moreover $F$ 
is totally real, it is known that the $D_{n}$ 's are bounded and $\lim _{n} D_{n}=(0)$ (see e.g. $[\mathrm{Gb}])$. Then:

\section{Theorem 1.3.}

(i) For any $n \geqslant 0$, we have a canonical surjection $H^{1}\left(G_{n}, U_{n}\right) \rightarrow H^{1}\left(G_{n}, U_{n}^{\prime}\right)$ whose kernel $N_{n}$ takes place in an exact sequence:

$0 \rightarrow \operatorname{Ker}\left(D_{0} \stackrel{\text { nat }}{\rightarrow} D_{n}\right) \rightarrow N_{n} \rightarrow\left(\mathbb{Z} / p^{n} \mathbb{Z}\right)^{s} \rightarrow \operatorname{Coker}\left(D_{0} \stackrel{\text { nat }}{\rightarrow} D_{n}\right) \rightarrow 0$.

If moreover $F$ is totally real, we have a natural surjection $H^{1}\left(\Gamma, U_{\infty}\right) \rightarrow$ $H^{1}\left(\Gamma, U_{\infty}^{\prime}\right)$ whose kernel $N_{\infty}$ takes place in an exact sequence:

$$
0 \rightarrow D_{0} \rightarrow N_{\infty} \rightarrow\left(\mathbb{Q}_{p} / \mathbb{Z}_{p}\right)^{s} \rightarrow 0 .
$$

(ii) For any $n \geqslant 0$, we have a canonical exact sequence:

$$
0 \rightarrow H^{2}\left(G_{n}, U_{n}\right) \rightarrow H^{2}\left(G_{n}, U_{n}^{\prime}\right) \rightarrow\left(\mathbb{Z} / p^{n} \mathbb{Z}\right)^{s} \rightarrow N_{n} \rightarrow 0 .
$$

If moreover $F$ is totally real, then for all $n \geqslant m>>0, H^{2}\left(G_{n, m}, U_{n}\right) \stackrel{\sim}{\rightarrow}$ $H^{2}\left(G_{n, m}, U_{n}^{\prime}\right), H^{2}\left(\Gamma_{m}, U_{\infty}\right) \stackrel{\sim}{\rightarrow} H^{2}\left(\Gamma_{m}, U_{\infty}^{\prime}\right)$ and $N_{\infty} \simeq\left(\mathbb{Q}_{p} / \mathbb{Z}_{p}\right)^{s}$. In particular, $N_{\infty}$ is the maximal divisible subgroup of $H^{1}\left(\Gamma, U_{\infty}\right)$.

Proof. Statement (i) is just theorem 3.1 of [N]. For the convenience of the reader, we repeat briefly the main argument. Denoting by $S_{n}$ the set of $p$-places of $F_{n}$ (of cardinal $s$ ), we get by class field theory a commutative diagram with exact rows and trivial $G_{n}$-action:

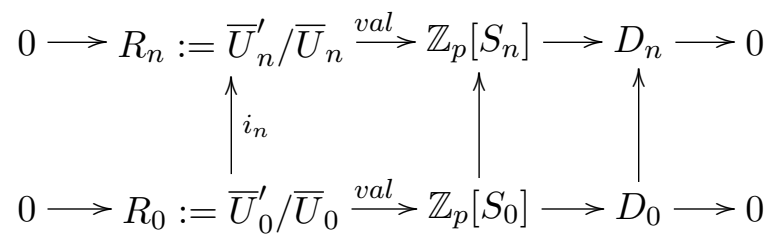

The tautological exact sequence $0 \rightarrow \bar{U}_{n} \rightarrow \bar{U}_{n}^{\prime} \rightarrow R_{n} \rightarrow 0$ gives by cohomology an exact sequence:

$$
0 \rightarrow N_{n}:=\text { Coker } i_{n} \rightarrow H^{1}\left(G_{n}, U_{n}\right) \rightarrow H^{1}\left(G_{n}, U_{n}^{\prime}\right) \rightarrow 0,
$$

and a simple diagram chase in (2) gives the desired description of $N_{n}$ (for details, see $[\mathrm{N}])$. Note that the factor $\left(\mathbb{Z} / p^{n} \mathbb{Z}\right)^{s}$ in statement (i) is just $H^{2}\left(G_{n}, \mathbb{Z}_{p}\left[S_{n}\right]\right)$. Let us next prove (ii). The exact hexagon of cohomology (since $G_{n}$ is cyclic)

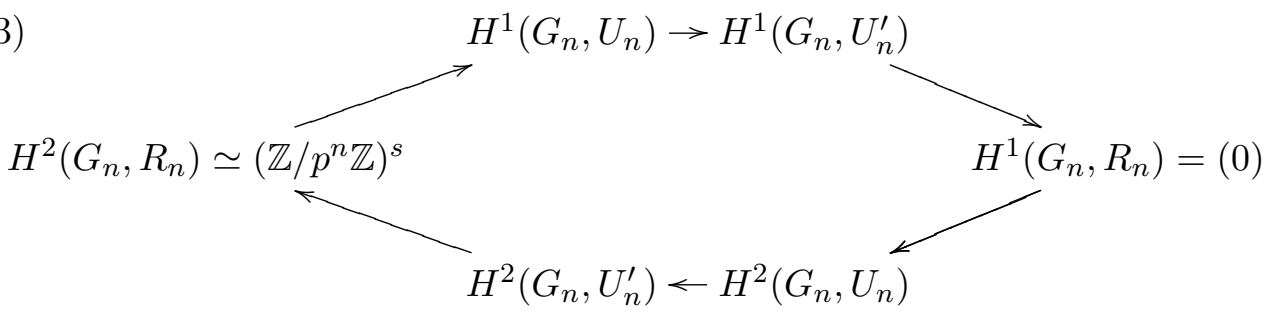


yields the first exact sequence announced in (ii).

Suppose now that $F$ is totally real. Then the $D_{n}$ 's are bounded; more precisely, there exists $n_{1} \geqslant 0$ such that for all $n \geqslant m \geqslant n_{1}, D_{n} \stackrel{\sim}{\rightarrow} D_{m}$ via norms (see e.g. [Gb]). Since $\mathbb{Z}_{p}\left[S_{n}\right] \stackrel{\text { norm }}{\rightarrow} \mathbb{Z}_{p}\left[S_{m}\right]$ by $(\mathrm{R})$, we also have $R_{n} \stackrel{\sim}{\rightarrow} R_{m}$ via norms. This means that the natural map $\hat{H}^{0}\left(G_{n, m}, U_{n}\right) \rightarrow \hat{H}^{0}\left(G_{n, m}, U_{n}^{\prime}\right)$ is surjective, hence $H^{2}\left(G_{n, m}, U_{n}\right) \stackrel{\sim}{\rightarrow} H^{2}\left(G_{n, m}, U_{n}^{\prime}\right)$ in the exact hexagon (3) above (replacing the 0 th level by the $m$ th). Taking inductive limits on $n$, we get $H^{2}\left(\Gamma_{m}, U_{\infty}\right) \stackrel{\sim}{\rightarrow} H^{2}\left(\Gamma_{m}, U_{\infty}^{\prime}\right)$ and $N_{\infty} \simeq\left(\mathbb{Q}_{p} / \mathbb{Z}_{p}\right)^{s}$. Since $H^{1}\left(\Gamma, U_{\infty}^{\prime}\right)$ is finite, the latter isomorphism implies that $N_{\infty}$ is the maximal divisible subgroup of $H^{1}\left(\Gamma, U_{\infty}\right)$, as desired.

$>$ From now on, we'll denote by $D_{\infty}$ the inverse $\operatorname{limit} \lim D_{n}$ w.r.t. norms. If $F$ is totally real, $D_{\infty}$ is finite, hence $D_{\infty} \subset X_{\infty}^{\text {tor }}$ (see [Gb]). In the general case, the norm maps $D_{n} \rightarrow D_{m}(n \geqslant m)$ are surjective, hence $D_{\infty}$ is trivial if and only if all the $D_{n}$ 's are trivial.

Corollary 1.4. Suppose that $D_{\infty}=(0)$. Then, for any $n \geqslant 0$, we have:

- an isomorphism of groups: $H^{1}\left(G_{n}, U_{n}\right) \simeq\left(\mathbb{Z} / p^{n} \mathbb{Z}\right)^{s} \oplus H^{1}\left(G_{n}, U_{n}^{\prime}\right)$;

- an isomorphism of Galois modules: $H^{2}\left(G_{n}, U_{n}\right) \simeq H^{2}\left(G_{n}, U_{n}^{\prime}\right)$.

Proof. Just apply theorem 1.3.

As noticed before, examples of fields for which $D_{\infty}=(0)$ are totally real fields $F$ such that $X_{\infty}(F)$ has no non-trivial finite submodule. Another class of examples is given by condition (A), thanks to the following result (first observed by J.-R. Belliard):

Lemma 1.5. For an abelian number field satisfying hypothesis (A), we have $A_{n}=$ $A_{n}^{\prime}$ for all $n \geqslant 0$.

Proof. Let $\mathbb{B}_{a}:=\mathbb{B}_{\infty} \cap F$ be the highest of the layers of the cyclotomic $\mathbb{Z}_{p}$-extension $\mathbb{B}_{\infty} / \mathbb{Q}$ of $\mathbb{Q}$ contained in $F$. To begin with, we show that $F / \mathbb{B}_{a}$ is tamely ramified at $p$. If $F$ is unramified at $p$, there is nothing to do. Otherwise the general idea comes from [LF], section 3: Let $f:=p^{a+r+1} m$ be the conductor of $F$ where $p \nmid m$ and $r \geqslant 0$. We have the following diagram: 


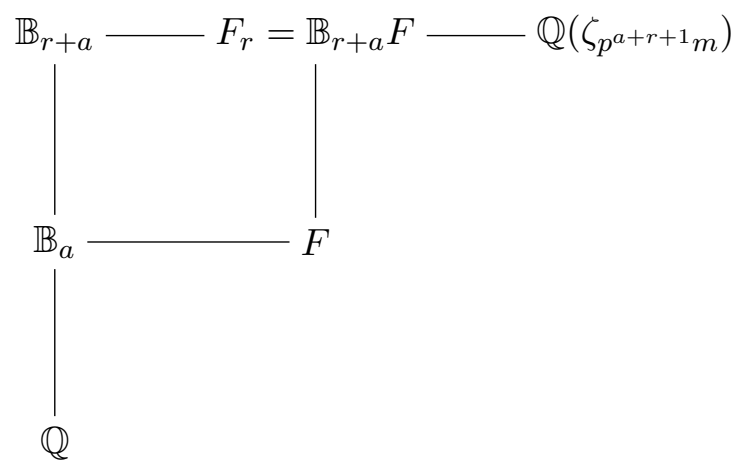

Because of $(\mathrm{A})$, the wild ramification index of $p$ in the extension $F_{r} / F$ is equal to its degree $\left[F_{r}: F\right]=p^{r}$. Consequently, the wild index ramification of $p$ in $F / \mathbb{B}_{a}$ is equal to 1 (since that of $p$ in $\mathbb{Q}\left(\zeta_{p^{a+r+1} m}\right) / \mathbb{Q}$ is $p^{a+r}$ ). Therefore $F / \mathbb{B}_{a}$ is tamely ramified at $p$ (actually we can even notice that, if $M$ denotes the field fixed by the wild inertia group of $p$ in $F / \mathbb{Q}$, then $F=M \mathbb{B}_{a}$ and $M$ is the unique number field which is tamely ramified at $p$ and has the same cyclotomic $\mathbb{Z}_{p}$-extension as $F$; this fact will be used later).

We then deduce that, for all $n \geqslant 0$, the extension $F_{n} / \mathbb{B}_{a+n}$ is tamely ramified at $p$, with ramification index $e_{n}$. For all $n \geqslant 0$, denote by $\mathfrak{p}_{n}$ the $p$-adic prime of $F_{n}$ and let $\pi_{n}$ be the norm of the element $1-\zeta_{p^{a+n+1}}$ in the extension $\mathbb{Q}\left(\zeta_{p^{a+n+1}}\right) / \mathbb{B}_{a+n}:$

$$
\pi_{n}=\mathrm{N}_{\mathbb{Q}\left(\zeta_{p^{a+n+1}}\right) / \mathbb{B}_{a+n}}\left(1-\zeta_{p^{a+n+1}}\right) .
$$

The $p$-adic prime of $\mathbb{B}_{a+n}$ being principal, generated by $\pi_{n}$, we have:

$$
\left(\pi_{n}\right)=\mathfrak{p}_{n}^{e_{n}} \text { with } p \nmid e_{n} .
$$

Thus the order of $\mathfrak{p}_{n}$ in the class group of $F_{n}$ is prime to $p$ and so $A_{n}^{\prime}=A_{n}$.

Let us gather in a single proposition all the cohomological statements - in addition to 1.4 - which can be derived under hypothesis (A):

Proposition 1.6. Let $F$ be an abelian number field satisfying hypothesis (A). Then we have:

(i) For all $n \geqslant 0$, for all $i \in \mathbb{Z}$, canonical isomorphisms:

$$
\hat{H}^{i-2}\left(G_{n}, A_{n}\right) \simeq \hat{H}^{i}\left(G_{n}, U_{n}^{\prime}\right) \simeq \hat{H}^{i}\left(G_{n},\left(X_{\infty}^{\text {tor }}\right)^{\Gamma_{n}}\right) .
$$

(ii) For all $n \geqslant 0$, a capitulation exact sequence:

$$
0 \rightarrow H^{1}\left(G_{n}, U_{n}^{\prime}\right) \rightarrow A_{0} \rightarrow A_{n}^{G_{n}} \rightarrow H^{2}\left(G_{n}, U_{n}^{\prime}\right) \rightarrow 0 .
$$

(iii) $\operatorname{Ker}\left(A_{0} \rightarrow A_{\infty}^{\Gamma}\right) \simeq H^{1}\left(\Gamma, U_{\infty}^{\prime}\right) \simeq\left(X_{\infty}^{\text {tor }}\right)_{\Gamma}$, and $\operatorname{Coker}\left(A_{0} \rightarrow A_{\infty}^{\Gamma}\right) \simeq H^{2}\left(\Gamma, U_{\infty}^{\prime}\right)=(0)$.

(iv) $H^{1}\left(\Gamma, U_{\infty}\right) \simeq \mathbb{Q}_{p} / \mathbb{Z}_{p} \oplus\left(X_{\infty}^{\text {tor }}\right)_{\Gamma}$ (as groups) and $H^{2}\left(\Gamma, U_{\infty}\right)=(0)$. 
Proof. (i) comes from lemma 1.2, knowing that, under $(\mathrm{A}), \hat{U}_{n}^{\prime}=\bar{U}_{n}^{\prime}$ and $\left(X_{\infty}\right)_{\Gamma_{n}} \stackrel{\sim}{\rightarrow}$ $A_{n}$.

(ii) comes from the tautological exact sequence of Tate cohomology groups

$$
0 \rightarrow \hat{H}^{-1}\left(G_{n}, A_{n}\right) \rightarrow\left(A_{n}\right)_{G_{n}} \stackrel{\text { norm }}{\rightarrow} A_{n}^{G_{n}} \rightarrow \hat{H}^{0}\left(G_{n}, A_{n}\right) \rightarrow 0,
$$

knowing that $\left(A_{n}\right)_{G_{n}} \simeq A_{0}$ under hypothesis (A).

(iii) comes from (i) and (ii) by taking inductive limits.

(iv) comes from 1.4 and (iii) by taking inductive limits.

\section{Cohomology of Circular units And Universal nORMS}

In this section, $F$ denotes a totally real abelian field. Let us recall Sinnott's definition of circular units of $F([\mathrm{~S}])$. Let $U_{F}$ (respectively $U_{F}^{\prime}$ ) denote the units (resp. $(p)$-units) of $F$. We define $C y c(F)$ to be the subgroup of $F^{\times}$generated by -1 and all elements $\mathrm{N}_{\mathbb{Q}\left(\zeta_{N}\right) / F \cap \mathbb{Q}\left(\zeta_{N}\right)}\left(1-\zeta_{N}^{a}\right)$, where $N>1$ and $N \nmid a$. The intersection $C y c(F) \cap U_{F}$ (respectively $C y c(F) \cap U_{F}^{\prime}$ ) is the group of circular units (resp. circular $(p)$-units) of $F$, denoted by $C(F)$ (resp. $C^{\prime}(F)$ ). Moreover, if $F_{n}$ is the $n$-th layer of the cyclotomic $\mathbb{Z}_{p}$-extension of $F$, we let $C_{n}$ (respectively $C_{n}^{\prime}$ ) denote the group $C\left(F_{n}\right)$ (resp. $C^{\prime}\left(F_{n}\right)$ ). The main interest of Sinnott's circular units in Iwasawa theory comes from Sinnott's index formula: up to powers of 2, $\left(U_{n}: C_{n}\right)$ " $=" c_{n} h_{n}$, where $h_{n}$ denotes the class number of $F_{n}$ and the $c_{n}$ 's are rational bounded constants. This implies that the orders of $A_{n}$ and $B_{n}:=\bar{U}_{n} / \bar{C}_{n}$ are asymptotically equivalent, but it is known that $A_{n}$ and $B_{n}$ are not isomorphic in general.

When the prime $p$ is unramified in the base field $F$, J.-R. Belliard has computed in [B1] the $G_{n}$-cohomology of $C_{n}$ under a (mildly) restrictive hypothesis called (HB) ([B1], p.34) which is satisfied in many "usual" cases. The appendix at the end of the present paper introduces a generalization (gHB) of hypothesis (HB), which allows to compute the $G_{n}$-cohomology of $C_{n}$. Actually, the condition (gHB) bears on the maximal subfield of $F_{\infty}$ in which $p$ is tamely ramified (appendix, definition 6.4). Our main hypothesis in the sequel will be a certain criterion (DG) on Galois descent for the circular units (see definition 2.1 below), which is a consequence of (gHB) but is probably not equivalent to it (although no counter-example is known).

Lemma-Definition 2.1. Let $\bar{C}_{\infty}:=\lim _{C_{n}}$. Assuming $(R)$, the following conditions are equivalent:

(i) The $\Lambda$-module $\bar{C}_{\infty}$ is free (of rank $r_{1}=[F: \mathbb{Q}]$ );

(ii) The $\bar{C}_{n}$ 's satisfy asymptotic Galois descent, i.e.

$$
\forall n \geqslant m>>0, \quad \bar{C}_{n}^{G_{n, m}} \simeq \bar{C}_{m}
$$


(iii) The $\bar{C}_{n}$ 's satisfy Galois descent, i.e.

$$
\forall n \geqslant m \geqslant 0, \quad \bar{C}_{n}^{G_{n, m}} \simeq \bar{C}_{m}
$$

In the sequel, following the notations of $[\mathrm{N}]$, we shall call $(D G)$ these equivalent properties (see also 3.2 below).

Proof. The conditions (i) and (ii) are equivalent by [B2], Théorème 2.2 (even without hypothesis $(\mathrm{R})$ ). For the equivalence with (iii), see $[\mathrm{N}]$, Corollaire 4.4.

In the sequel, let $\widetilde{C}_{n}$ denote the image of the natural homomorphism $\bar{C}_{\infty} \rightarrow \bar{C}_{n}$. A classical argument of compacity shows that $\widetilde{C}_{n}=\underset{m \geqslant n}{\cap} \mathrm{N}_{F_{m} / F_{n}}\left(\bar{C}_{m}\right)$, which explains why the elements of $\widetilde{C}_{n}$ are referred to as universal norms. Then:

Lemma-Definition 2.2. Under $(D G)$, the module $\Phi:=\bar{C}_{0} / \widetilde{C}_{0}$ is isomorphic by extension to $\bar{C}_{n} / \widetilde{C}_{n}$ for all $n \geqslant 0$. The $\mathbb{Z}_{p}$-rank of $\Phi$ is $(s-1)$, where $s$ is the number of $p$-adic primes of $F$.

Proof. See $[\mathrm{N}]$, lemme 4.5. The key point is a technical lemma based on distribution relations ([B2], lemme 2.5) which shows that $\bar{C}_{n}=\widetilde{C}_{n} \bar{C}^{i n}$, where $C^{i n}$ denotes the group of circular units of the subfield $F^{i n}$ of inertia at $p$.

$>$ From now on, let us write $t \Phi$ for the $\mathbb{Z}_{p}$-torsion submodule of $\Phi$ and $p^{n}(t \Phi)$ for the $p^{n}$-torsion submodule. Then:

Theorem 2.3. Under ( $D G)$, for all $n \geqslant m \geqslant 0$, we have an isomorphism of Galois modules

$$
H^{2}\left(G_{n, m}, C_{n}\right) \simeq t \Phi / p^{n-m} \oplus\left(\mathbb{Z} / p^{n-m} \mathbb{Z}\right)^{s-1},
$$

and an exact sequence:

$$
0 \rightarrow\left(\mathbb{Z} / p^{n-m} \mathbb{Z}\right)^{s} \rightarrow H^{1}\left(G_{n, m}, C_{n}\right) \rightarrow{ }_{p^{n-m}}(t \Phi) \rightarrow 0 .
$$

At infinite level, writing $C_{\infty}:=\lim _{\longrightarrow} C_{n}$, we have isomorphisms :

$H^{2}\left(\Gamma, C_{\infty}\right) \simeq\left(\mathbb{Q}_{p} / \mathbb{Z}_{p}\right)^{s-1}$ (as modules) and $H^{1}\left(\Gamma, C_{\infty}\right) \simeq t \Phi \oplus\left(\mathbb{Q}_{p} / \mathbb{Z}_{p}\right)^{s}$ (as groups).

Proof. This is [N], théorème 4.6; if $p$ is unramified in $F$, see also [B1], proposition 3.15. For later use, we must indicate that the factor $\left(\mathbb{Z} / p^{n-m} \mathbb{Z}\right)^{s}$ comes from the $G_{n, m^{-}}$-cohomology of $\widetilde{C}_{n}$, actually from a canonical exact sequence

$$
0 \rightarrow H^{1}\left(G_{n, m}, \widetilde{C}_{n}\right) \simeq\left(\mathbb{Z} / p^{n-m} \mathbb{Z}\right)^{s} \rightarrow H^{1}\left(G_{n, m}, \bar{C}_{n}\right) \rightarrow H^{1}\left(G_{n, m}, \Phi\right) \simeq{ }_{p^{n-m}}(t \Phi) \rightarrow 0 .
$$

([N], lemme 4.2). 
Of course, theorem 2.3 is of interest only if we can produce "usual" examples of fields $F$ satisfying (DG). Let $F^{\text {tam }}$ be the maximal subfield of $F$ in which $p$ is tamely ramified. It is known that $\left(F^{\operatorname{tam}}\right)_{\infty}=F_{\infty}$ ([B2], lemme 1.2 ; see also 1.5 above). Since (DG) is an asymptotic condition (lemma 2.1 (ii)), we can replace $F$ by $F^{t a m}$ if necessary. Then (gHB) implies (DG) (appendix, theorem 6.6). In particular:

Proposition 2.4. F satisfies (DG) if one of the following conditions holds:
(a) $p \nmid[F: \mathbb{Q}]$;
(b) $p$ is inert in $F^{\text {in }}$;
(c) $s=1$.

Proof. We can suppose that $F=F^{\text {tam }}$, so that we have the following diagram of field extensions

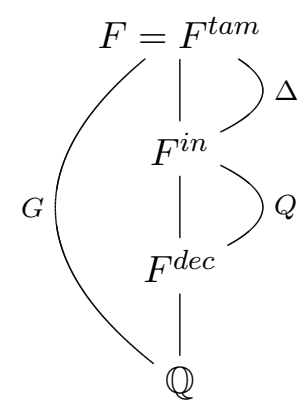

where $F^{i n}$ and $F^{d e c}$ are the subfields of inertia and of decomposition at $p$. Then $\Delta$ is of order prime to $p$ and $Q$ is cyclic, generated by the Frobenius $\sigma_{p}$ at $p$. In all three cases (a), (b), (c), the Sylow $p$-subgroup (or $p$-quotient) of $G=\operatorname{Gal}(F / \mathbb{Q}$ ) is cyclic, and proposition 6.5.2 of the appendix applies, i.e. condition (gHB) is satisfied.

Remark: In the semi-simple case (condition (a)), we could also have proceeded directly by calculating $\bar{C}_{\infty}$ characterwise, using Coleman's theory ([T], 6.2).

Before proceeding further, let us give a more explicit description of $\Phi$ and $t \Phi$. We adopt the notations in the proof of 2.4: $F^{\text {tam }}, F^{i n}, F^{\text {dec }}, \Delta=\operatorname{Gal}\left(F^{t a m} / F^{i n}\right)$, $Q=<\sigma_{p}>=\operatorname{Gal}\left(F^{\text {in }} / F^{\text {dec }}\right)$. Let us write $C^{\text {in }}:=C\left(F^{\text {in }}\right), C^{\text {dec }}:=C\left(F^{\text {dec }}\right), D^{\text {in }}$ : $=C y c\left(F^{i n}\right)$, and also $\bar{C}^{i n}:=\overline{C^{i n}}, \bar{C}^{d e c}:=\overline{C^{d e c}}, \bar{D}^{i n}:=\overline{D^{i n}}$. Then:

Proposition 2.5. Under (R), we have:

(i) $\Phi \simeq \bar{C}^{i n} /\left(\bar{D}^{i n}\right)^{\sigma_{p}-1}$, if $p$ is tamely ramified in $F$.

(ii) $t \Phi \simeq \hat{H}^{-1}\left(Q, \bar{C}^{i n}\right)$. 
Proof. (i) We refer to the diagram of extensions in 2.4, supposing that $F=F^{\text {tam }}$. Since $\bar{C}_{0}=\widetilde{C}_{0} \bar{C}^{i n}$, we have $\Phi \simeq \bar{C}^{i n} / \widetilde{C}_{0} \cap \bar{C}^{i n}$. But $\widetilde{C}_{0} \cap \bar{C}^{i n}=\left(\widetilde{C}_{0}\right)^{\Delta}$, and since $\Delta$ is of order prime to $p$, we have $\left(\widetilde{C}_{0}\right)^{\Delta}=\mathrm{N}_{\Delta}\left(\widetilde{C}_{0}\right)$, where $\mathrm{N}_{\Delta}$ is the norm map of $F / F^{i n}$. Again by using the distribution relations, we know that $\mathrm{N}_{\Delta}\left(\widetilde{C}_{0}\right)=\left(\bar{D}^{i n}\right)^{\sigma_{p}-1}$ (for further details, see [B2]), and therefore

$$
\Phi \simeq \bar{C}^{i n} /\left(\bar{D}^{i n}\right)^{\sigma_{p}-1} .
$$

(ii) To compute $t \Phi$, we first notice that $t \Phi$ depends only on $F^{t a m}$ (theorem 2.3) so that we can take $F=F^{\text {tam }}$. Apply then the norm $\mathrm{N}_{Q}$ from $F^{i n}$ to $F^{\text {dec: }}$ : $\bar{C}^{i n} /\left(\bar{D}^{i n}\right)^{\sigma_{p}-1} \stackrel{\mathrm{N}_{Q}}{\longrightarrow} \bar{C}^{\text {dec }}$. Since $\bar{C}^{\text {dec }}$ is $\mathbb{Z}_{p}$-torsion-free $(p \neq 2)$, we deduce that $t \Phi$ is the kernel of that norm, namely

$$
t \Phi \simeq \hat{H}^{-1}\left(Q, \bar{C}^{i n}\right) .
$$

Corollary 2.6. The triviality of $t \Phi$ is implied by one of the following conditions:

(a) $p$ does not divide the order of the Frobenius $\sigma_{p}$;

(b) $s=1$ and the conductor of $F^{\text {in }}$ is a power of a single prime $\ell$ (necessarily $\ell \neq p)$.

Proof. (a) If $p \nmid\left[F^{i n}: F^{d e c}\right]$, this means that $Q$ is of order prime to $p$ and hence $\hat{H}^{-1}\left(Q, \bar{C}^{i n}\right)=(0)$.

(b) If $s=1, \Phi=t \Phi$ and we can apply 2.5 (i) to kill $\Phi$. For all $m \geqslant 0$, let $\epsilon_{m}=\mathrm{N}_{\mathbb{Q}\left(\zeta_{m}\right) / \mathbb{Q}\left(\zeta_{m}\right) \cap F^{i n}}\left(1-\zeta_{m}\right)$. It follows from the definitions that

$$
C^{i n}=<\left\{\epsilon_{m}, m \text { composed, } m \mid \operatorname{cond}\left(F^{i n}\right)\right\} \cup\left\{\epsilon_{m}^{\sigma_{p}-1}, m \text { a power of a prime } \ell\right\}>
$$

and

$$
\left(D^{i n}\right)^{\sigma_{p}-1}=<\left\{\epsilon_{m}^{\sigma_{p}-1}, m \geqslant 0\right\}>.
$$

It appears that if moreover $\operatorname{cond}\left(F^{i n}\right)$ is not composed, then $\Phi=(0)$.

Under hypothesis (A) (which implies (DG) by 2.4), we can get information on the cohomology of the circular $(p)$-units:

Lemma 2.7. Under (A), for all $n \geqslant 0, \quad \bar{C}_{n}^{\prime} / \bar{C}_{n} \stackrel{\sim}{\rightarrow} \bar{U}_{n}^{\prime} / \bar{U}_{n}$ and $\bar{U}_{n} / \bar{C}_{n} \stackrel{\sim}{\rightarrow}$ $\bar{U}_{n}^{\prime} / \bar{C}_{n}^{\prime}$.

Proof. It obviously suffices to show the first isomorphism. We proceed almost as in the proof of lemma 1.5 , replacing $\pi_{n}$ by an element $\varpi_{n}$ defined as follows:

$$
\varpi_{n}=\mathrm{N}_{\mathbb{Q}\left(\zeta_{p^{a+n+1}}\right) / F_{n} \cap \mathbb{Q}\left(\zeta_{p^{a+n+1}}\right)}\left(1-\zeta_{p^{a+n+1}}\right) \in C_{n}^{\prime} .
$$


The situation is the following

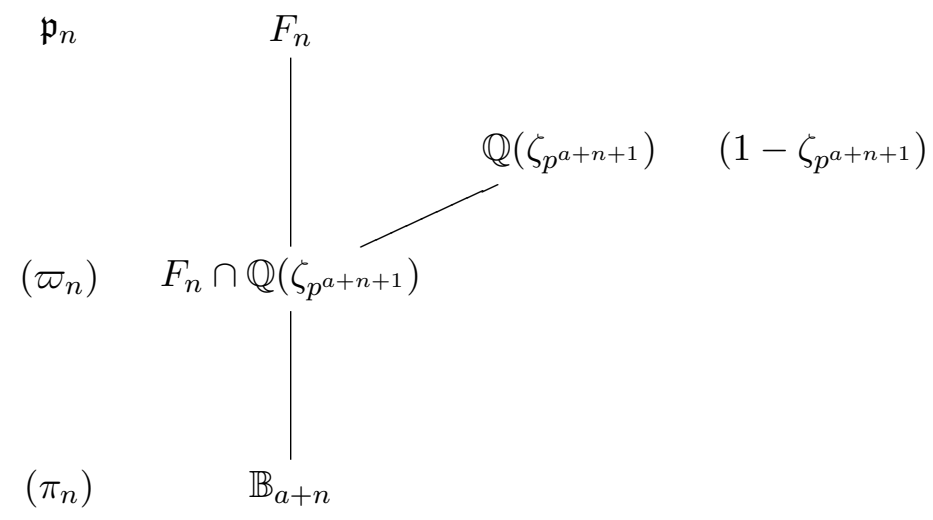

The unique prime of $F_{n} \cap \mathbb{Q}\left(\zeta_{p^{a+n+1}}\right)$ above $p$ is the ideal $\left(\varpi_{n}\right)$. Since $F_{n} / \mathbb{B}_{a+n}$ is tamely ramified at $p$, we can write $\left(\varpi_{n}\right)=\mathfrak{p}_{n}^{e_{n}}$ with $p \nmid e_{n}$. As a result, the element $\hat{\varpi}_{n}:=\varpi_{n} \otimes \frac{1}{e_{n}} \in \bar{C}_{n}^{\prime}$ satisfies at the same time $\bar{C}_{n}^{\prime}=\bar{C}_{n} \oplus \mathbb{Z}_{p} \hat{\varpi}_{n}$ and $\bar{U}_{n}^{\prime}=\bar{U}_{n} \oplus \mathbb{Z}_{p} \hat{\varpi}_{n}$, whence the result.

Proposition 2.8. Under (A), for all $n \geqslant 0, \hat{H}^{0}\left(G_{n}, C_{n}^{\prime}\right) \simeq t \Phi / p^{n}$ (as Galois modules) and $H^{1}\left(G_{n}, C_{n}^{\prime}\right) \simeq{ }_{p^{n}} t \Phi$ (as groups). In particular, the triviality of $t \Phi$ implies the $G_{n}$-cohomological triviality of all the $C_{n}^{\prime}$ 's.

Proof. We have seen in the proof of 1.3 that the triviality of the $D_{n}$ 's (which is a consequence of $(\mathrm{A}))$ implies $\hat{H}^{0}\left(G_{n}, U_{n}\right) \simeq \hat{H}^{0}\left(G_{n}, U_{n}^{\prime}\right)$, hence $\hat{H}^{0}\left(G_{n}, C_{n}\right) \simeq$ $\hat{H}^{0}\left(G_{n}, C_{n}^{\prime}\right)$ by 2.7. Exactly as in 1.3 , the exact hexagon of cohomology associated to the exact sequence $0 \rightarrow \bar{C}_{n} \rightarrow \bar{C}_{n}^{\prime} \stackrel{\text { val }}{\longrightarrow} \mathbb{Z}_{p} \rightarrow 0$ then breaks down to yield

$$
0 \rightarrow \mathbb{Z} / p^{n} \mathbb{Z} \rightarrow H^{1}\left(G_{n}, C_{n}\right) \rightarrow H^{1}\left(G_{n}, C_{n}^{\prime}\right) \rightarrow 0 .
$$

But $\mathbb{Z} / p^{n} \mathbb{Z}$ is injective, hence an isomorphism of groups: $H^{1}\left(G_{n}, C_{n}\right) \simeq H^{1}\left(G_{n}, C_{n}^{\prime}\right) \oplus$ $\mathbb{Z} / p^{n} \mathbb{Z}$. We then apply theorem 2.3 .

We are now ready to perform descent and co-descent. Of course, the two problems are not independent (see 3.7 and 4.5), and our arbitrary distinction between them is just for the sake of clarity of exposition.

\section{Descent For Units MOdUlo CIRCUlar Units}

In this section, $F$ is a totally real abelian field, $B_{n}:=\bar{U}_{n} / \bar{C}_{n}=$ the $p$-part of $U_{n} / C_{n}$, and $B_{\infty}=\lim _{\longrightarrow} B_{n}$ (with transition maps induced by inclusion). We aim to study the kernels and cokernels of the natural extension maps $j_{n}: B_{n} \rightarrow\left(B_{\infty}\right)^{\Gamma_{n}}$.

Theorem 3.1. The kernels $\operatorname{Ker} j_{n}$ stabilize (w.r.t. norms) and their inverse limit, $K N_{F}$ say, is equal to the maximal finite submodule $Y_{\infty}^{\text {tor }}$ of $Y_{\infty}=\lim _{\longleftarrow} B_{n}$. 
Proof. This is proposition 2.3 of [BN2], where $\varliminf_{\lim } \operatorname{Ker} j_{n}$ is called the "KuceraNekovar kernel" $K N_{F}$ associated to $F$.

The kernel $K N_{F}$ is an obstruction to condition (DG) in the following sense:

Corollary 3.2. The following are equivalent:

(i) $K N_{F}=(0)$;

(ii) $(D G)$ holds;

(iii) $\left(Y_{\infty}\right)^{\Gamma_{n}}=(0), \forall n \geqslant 0$.

Proof. According to 1.(b), the $\Lambda$-module $\bar{U}_{\infty}$ is free, hence $\bar{C}_{\infty}$ is $\Lambda$-free (i.e. condition (DG) holds) if and only if $Y_{\infty}=\bar{U}_{\infty} / \bar{C}_{\infty}$ has no non trivial finite submodule. By 3.1, this is equivalent to the vanishing of $K N_{F}$. The equivalence with (iii) is a consequence of the Main Conjecture: $Y_{\infty}$ has the same characteristic series as $X_{\infty}$, hence Leopoldt's conjecture for $F_{n}$ means that $\left(Y_{\infty}\right)^{\Gamma_{n}}$ is finite or, equivalently, $\left(Y_{\infty}\right)^{\Gamma_{n}}=\left(Y_{\infty}^{\text {tor }}\right)^{\Gamma_{n}}$.

As for the cokernels Coker $j_{n}$, we have a first obvious cohomological characterization (cp. 1.1 (i) and 1.6 (ii)):

Lemma 3.3. For all $n \geqslant m \geqslant 0$, let $j_{n, m}: B_{m} \rightarrow\left(B_{n}\right)^{G_{n, m}}$ be the canonical extension map. Then

$$
\text { Coker } j_{n, m} \simeq \operatorname{Ker}\left(H^{1}\left(G_{n, m}, C_{n}\right) \stackrel{n a t}{\rightarrow} H^{1}\left(G_{n, m}, U_{n}\right)\right)
$$

and

$$
\text { Coker } j_{m} \simeq \operatorname{Ker}\left(H^{1}\left(\Gamma_{m}, C_{\infty}\right) \rightarrow H^{1}\left(\Gamma_{m}, U_{\infty}\right)\right),
$$

where $C_{\infty}:=\lim _{\longrightarrow} C_{n}$ and $U_{\infty}:=\lim _{\longrightarrow} U_{n}$.

Proof. It suffices to consider the following diagram with exact rows:

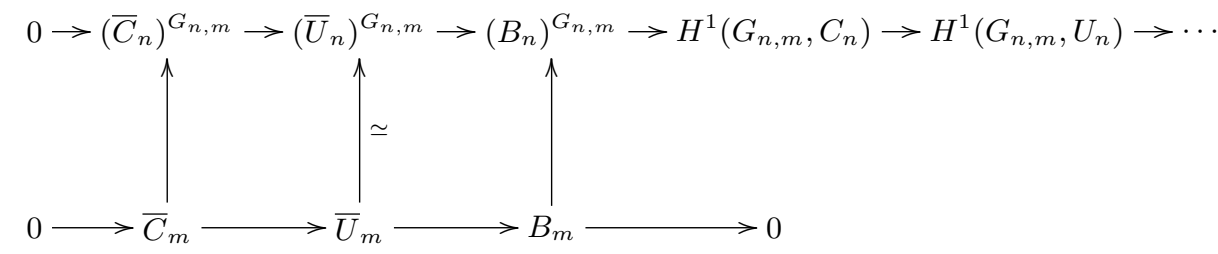

The point now is to give an amenable description of the kernel

$$
\operatorname{Ker}\left(H^{1}\left(\Gamma_{m}, C_{\infty}\right) \rightarrow H^{1}\left(\Gamma_{m}, U_{\infty}\right)\right) .
$$

We need to introduce a few (module theoretic) invariants attached to $F_{\infty} / F$. For all $n \geqslant 0$, let us define $Q_{n}:=\bar{U}_{n}^{\prime} / \bar{C}_{n}, Q_{\infty}=\lim Q_{n}$ (w.r.t. norms), $R_{n}:=\bar{U}_{n}^{\prime} / \bar{U}_{n}$, $R_{\infty}=\lim R_{n}$ (also w.r.t. norms). We have seen in the proof of 1.3 that $G_{n}$ acts 
trivially on $R_{n}$ and that $R_{\infty} \stackrel{\text { norm }}{\rightarrow} R_{n}$ for $n \geqslant 0$; if needed, we'll identify $R_{n}$ and $\left(R_{\infty}\right)^{\Gamma_{n}}$ (then $\Gamma_{n}$ will just serve as an index).

Lemma 3.4. For all $n \geqslant 0,\left(Q_{\infty}\right)^{\Gamma_{n}}$ (respectively $\left.\left(R_{\infty}\right)^{\Gamma_{n}}\right)$ is isomorphic to the kernel of the natural map $\left(\bar{C}_{\infty}\right)_{\Gamma_{n}} \rightarrow \bar{C}_{n}$ (respectively $\left(\bar{U}_{\infty}\right)_{\Gamma_{n}} \rightarrow \bar{U}_{n}$ ).

Proof. (see also [BN2], [N])

The tautological exact sequence $0 \rightarrow \bar{C}_{\infty} \rightarrow \bar{U}_{\infty}^{\prime} \rightarrow Q_{\infty} \rightarrow 0$ gives by descent:

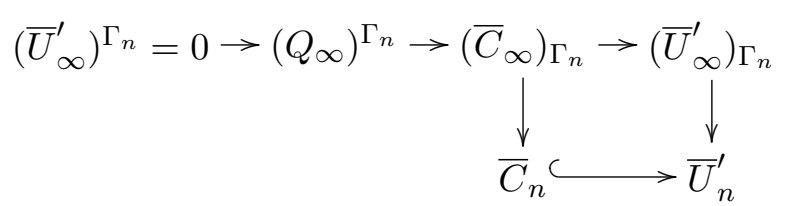

But the right-hand vertical map is injective by Kuz'min's result 1.(c). This yields the first announced isomorphism. The second is proved exactly in the same way.

$>$ From now on, let us denote by $\widetilde{U}_{n}$ the group of universal norms of units, i.e. $\widetilde{U}_{n}=\operatorname{Im}\left(\left(\bar{U}_{\infty}\right)_{\Gamma_{n}} \rightarrow \bar{U}_{n}\right)$. We introduce a co-descent module $\Psi$ which will intervene in the unwinding of the cokernels Coker $j_{n}$, thus showing the interdependence between the two problems (descent and co-descent).

Lemma-Definition 3.5. For any $n \geqslant 0$, put $\Psi_{n}:=\operatorname{Coker}\left(\left(Q_{\infty}\right)^{\Gamma_{n}} \stackrel{\text { nat }}{\rightarrow}\left(R_{\infty}\right)^{\Gamma_{n}}\right)$. The $\Psi_{n}$ 's are $G_{n}$-trivial finite modules which stabilize w.r.t. inclusion maps. Define $\Psi:=\lim _{\longrightarrow} \Psi_{n}$ (finite).

$$
\operatorname{Under}(D G), \Psi_{n} \simeq \operatorname{Ker}\left(\left(Y_{\infty}\right)_{\Gamma_{n}} \rightarrow \widetilde{U}_{n} / \widetilde{C}_{n}\right)
$$

Proof. The tautological exact sequence

$$
0 \rightarrow Y_{\infty}=\bar{U}_{\infty} / \bar{C}_{\infty} \rightarrow Q_{\infty}=\bar{U}_{\infty}^{\prime} / \bar{C}_{\infty} \rightarrow R_{\infty}=\bar{U}_{\infty}^{\prime} / \bar{U}_{\infty} \rightarrow 0
$$

gives by descent:

$$
0 \rightarrow\left(Y_{\infty}\right)^{\Gamma_{n}} \rightarrow\left(Q_{\infty}\right)^{\Gamma_{n}} \rightarrow\left(R_{\infty}\right)^{\Gamma_{n}} \longrightarrow\left(Y_{\infty}\right)_{\Gamma_{n}} \rightarrow \cdots
$$


By Leopoldt's conjecture for $F_{n},\left(Y_{\infty}\right)_{\Gamma_{n}}$ is finite and so is $\Psi_{n}$. The $\Psi_{n}$ 's obviously stabilize by noetherianity. Under (DG), the commutative diagram

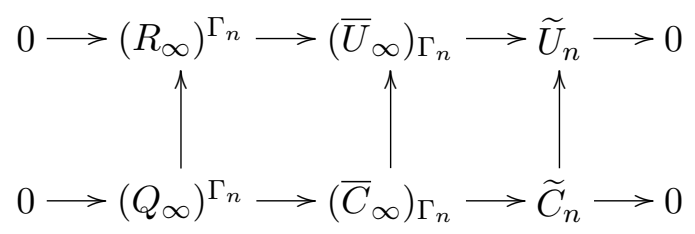

immediately gives the isomorphism $\Psi_{n} \simeq \operatorname{Ker}\left(\left(Y_{\infty}\right)_{\Gamma_{n}} \rightarrow \widetilde{U}_{n} / \widetilde{C}_{n}\right)$.

Now we can give a cohomological characterization of $\Psi_{n}$ :

Lemma 3.6. Under (DG), $\Psi_{m}$ is canonically isomorphic to

$$
\operatorname{Ker}\left(\operatorname{Div} H^{1}\left(\Gamma_{m}, C_{\infty}\right) \rightarrow \operatorname{Div} H^{1}\left(\Gamma_{m}, U_{\infty}\right)\right),
$$

for any $m>0$. Here $\operatorname{Div}(\cdot)$ denotes the maximal divisible subgroup.

Proof. Under (DG), $\left(Y_{\infty}\right)^{\Gamma_{n}}=(0)$ by 3.2 , hence the exact sequence (4) becomes: $0 \rightarrow\left(Q_{\infty}\right)^{\Gamma_{n}} \rightarrow\left(R_{\infty}\right)^{\Gamma_{n}} \rightarrow \Psi_{n} \rightarrow 0$. Taking $G_{n^{-}}$cohomology, we get:

$$
\begin{aligned}
\cdots \rightarrow H^{1}\left(G_{n},\left(R_{\infty}\right)^{\Gamma_{n}}\right) \simeq \operatorname{Hom}\left(G_{n}, \mathbb{Z}_{p}^{s}\right) & =0 \rightarrow H^{1}\left(G_{n}, \Psi_{n}\right)=\operatorname{Hom}\left(G_{n}, \Psi_{n}\right) \simeq p^{n} \Psi_{n} \\
\rightarrow H^{2}\left(G_{n},\left(Q_{\infty}\right)^{\Gamma_{n}}\right) & \rightarrow H^{2}\left(G_{n},\left(R_{\infty}\right)^{\Gamma_{n}}\right) \rightarrow \cdots
\end{aligned}
$$

Fixing $m \geqslant 0$ and taking inductive limits on $n \geqslant m$, we get an isomorphism $\Psi_{m} \simeq \operatorname{Ker}\left(H^{2}\left(\Gamma_{m},\left(Q_{\infty}\right)^{\Gamma_{m}}\right) \rightarrow H^{2}\left(\Gamma_{m},\left(R_{\infty}\right)^{\Gamma_{m}}\right)\right)$. It remains to compute this kernel in terms of maximal divisible subgroups.

For any $n \geqslant m$, the exact sequence $0 \rightarrow\left(Q_{\infty}\right)^{\Gamma_{n}} \rightarrow\left(\bar{C}_{\infty}\right)_{\Gamma_{n}} \rightarrow \widetilde{C}_{n} \rightarrow 0$ (see the proof of 3.4) shows that $H^{1}\left(G_{n, m}, \widetilde{C}_{n}\right) \simeq H^{2}\left(G_{n, m},\left(Q_{\infty}\right)^{\Gamma_{n}}\right)$. But we have seen, in the proof of theorem 2.3, canonical exact sequences $0 \rightarrow H^{1}\left(G_{n, m}, \widetilde{C}_{n}\right) \rightarrow$ $H^{1}\left(G_{n, m}, \bar{C}_{n}\right) \rightarrow p^{n-m} t \Phi \rightarrow 0$ which give, on taking inductive limits over $n$, an exact sequence: $0 \rightarrow\left(\mathbb{Q}_{p} / \mathbb{Z}_{p}\right)^{s} \rightarrow H^{1}\left(\Gamma_{m}, C_{\infty}\right) \rightarrow t \Phi \rightarrow 0$. Since $t \Phi$ is finite, this shows that $H^{2}\left(\Gamma_{m},\left(Q_{\infty}\right)^{\Gamma_{m}}\right) \simeq \operatorname{Div} H^{1}\left(\Gamma_{m}, C_{\infty}\right)$ for any $m \geqslant 0$.

Similarly, we have seen, in the proof of theorem 1.3, canonical exact sequences

$$
0 \rightarrow H^{2}\left(G_{n, m}, R_{n}\right) \rightarrow H^{1}\left(G_{n, m}, U_{n}\right) \rightarrow H^{1}\left(G_{n, m}, U_{n}^{\prime}\right) \rightarrow 0
$$

for $n \geqslant m>>0$, which give, on taking inductive limits over $n$, an isomorphism $H^{2}\left(\Gamma_{m},\left(R_{\infty}\right)^{\Gamma_{m}}\right) \simeq \operatorname{Div} H^{1}\left(\Gamma_{m}, U_{\infty}\right)$, as desired.

We are now in a position to prove our main result for descent of unit class groups, namely: 
Theorem 3.7. Under $(D G)$, for all $m \geqslant 0$, the maps $j_{m}: B_{m} \rightarrow\left(B_{\infty}\right)^{\Gamma_{m}}$ are injective and their cokernels stabilize w.r.t. extension maps. For all $m>>0$, we have a canonical exact sequence (where $\widehat{(\cdot)}$ denotes Pontryagin duality):

$$
0 \rightarrow \Psi \rightarrow \text { Coker } j_{m} \rightarrow t \Phi \stackrel{\alpha}{\rightarrow} X_{\infty}^{\prime \text { tor }} \rightarrow\left(\widehat{{\widehat{B_{\infty}}}^{\text {tor }}}\right) \simeq B_{\infty} / \operatorname{Div}\left(B_{\infty}\right)
$$

Proof. Fix $m$ large enough and consider the following commutative diagram:

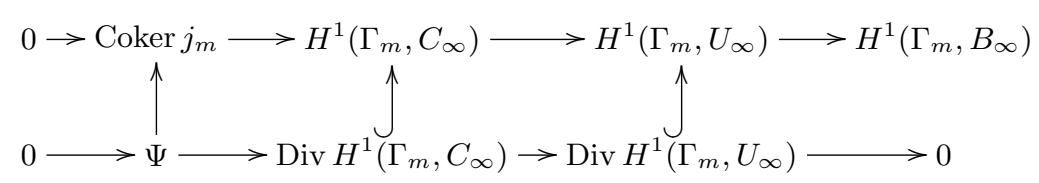

Applying 1.3 (ii), 2.3 and the snake lemma, we immediately get, for $m>>0$ :

$$
0 \rightarrow \Psi \rightarrow \text { Coker } j_{m} \rightarrow t \Phi \stackrel{\alpha}{\rightarrow} X_{\infty}^{\prime \text { tor }} \rightarrow H^{1}\left(\Gamma_{m}, B_{\infty}\right)
$$

Since $B_{\infty}$ is torsion, $H^{1}\left(\Gamma_{m}, B_{\infty}\right) \simeq\left(B_{\infty}\right)_{\Gamma_{m}}$ hence, by duality, $\left.H^{1} \widehat{\left(\Gamma_{m}, B_{\infty}\right.}\right) \simeq$ $\left(\widehat{B_{\infty}}\right)^{\Gamma_{m}}$. But it is known that $H^{1}\left(\Gamma_{m}, B_{\infty}\right)$ is finite $([\mathrm{N}], 5.1)$, hence $\left(\widehat{B_{\infty}}\right)^{\Gamma_{m}}=$ $\left(\widehat{B}_{\infty}^{t o r}\right)^{\Gamma_{m}}=\widehat{B}_{\infty}^{\text {tor }}$ for $m>>0$.

\section{Remarks:}

1) In theorem $3.7,\left(\widehat{\widehat{B}_{\infty}^{\text {tor }}}\right)$ must be considered as a parameter, in the same way as $X_{\infty}^{\prime}{ }^{\text {tor }}$. Going back to the proof of lemma 3.3 , we can see readily that the cokernel of the map $X_{\infty}^{\prime \text { tor }} \rightarrow\left(\widehat{\widehat{B}_{\infty}^{\text {tor }}}\right)$ in 3.7 is isomorphic to

$\operatorname{Ker}\left(H^{2}\left(\Gamma_{m}, C_{\infty}\right) \rightarrow H^{2}\left(\Gamma_{m}, U_{\infty}\right)\right)$ for $m>>0$, but we miss a noetherian interpretation in the style of 3.5. See however the remarks after corollary 4.6 below.

2) In the unit class setting, Greenberg's conjecture asserts that the $B_{n}$ 's are bounded. It follows easily, under (DG), that Coker $j_{n}=(0)$ for $n>>0$, hence we must expect that $\Psi$ is trivial modulo (DG).

Corollary 3.8. Suppose moreover that $t \Phi=\Psi=(0)$. Then the $B_{n}$ 's satisfy asymptotic Galois descent, i.e. $B_{n}^{G_{n, m}} \simeq B_{m}, \forall n \geq m>>0$.

Proof. By 3.7, our hypotheses imply that $B_{m} \stackrel{\sim}{\longrightarrow} B_{\infty}^{\Gamma_{m}}$ for all $m>>0$, hence the corollary.

Without Greenberg's conjecture, the ambiguities in theorem 3.7 can be removed in case $(\mathrm{A})$ : 
Proposition 3.9. Under (A), for any $m \geqslant 0$, we have an exact sequence of abelian groups:

$$
0 \rightarrow \text { Coker } j_{m} \rightarrow t \Phi \rightarrow\left(X_{\infty}^{\text {tor }}\right)_{\Gamma_{m}} \rightarrow\left(\widehat{\widehat{B}_{\infty}^{\text {tor }}}\right)^{\Gamma_{m}} \rightarrow 0 .
$$

In particular, $\Psi_{m}=(0)$.

Proof. In case (A), we have seen that $B_{n} \simeq \bar{U}_{n}^{\prime} / \bar{C}_{n}^{\prime}$ (lemma 2.7). Repeating the argument of 3.7 , we get, for $n \geqslant m \geqslant 0$ :

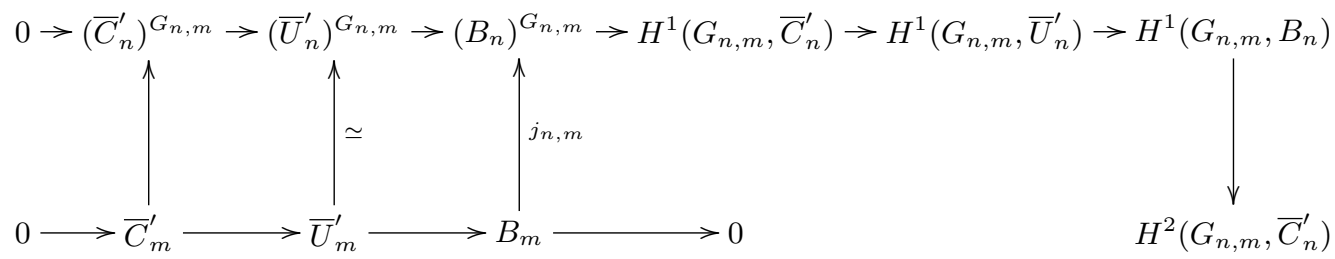

Applying 1.6, 2.8 and taking inductive limits over $n$, we immediately get the desired result.

Corollary 3.10. If $F$ satisfies $(A)$ and $t \Phi=(0)$, then:

(i) The $B_{n}$ 's satisfy Galois descent, i.e. $\left(B_{n}\right)^{G_{n, m}} \simeq B_{m}, \forall n \geqslant m \geqslant 0$

(ii) $\left(B_{\infty}\right)^{\Gamma} \simeq B_{0}$ and $\left(B_{\infty}\right)_{\Gamma} \simeq\left(X_{\infty}^{\text {tor }}\right)_{\Gamma}$ (cp. [O.I], proposition 1)

(iii) $\widehat{X_{\infty}^{\text {tor }}} \simeq{\widehat{B_{\infty}}}^{\text {tor }}$ (cp. [O.II], theorem 3)

(iv) $\forall i \in \mathbb{Z}, \forall n \geqslant m \geqslant 0, \hat{H}^{i}\left(G_{n, m}, B_{n}\right) \simeq \hat{H}^{i-2}\left(G_{n, m}, A_{n}\right)$ (canonically).

NB: a few remarks on the hypotheses in [O.I] and [O.II] are in order. Ozaki's hypothesis $(\mathrm{C})$, introduced in [O.I], is divided in three conditions $(\mathrm{C} 1),(\mathrm{C} 2)$ and (C3). Condition $(\mathrm{C} 1)$ is equivalent to $(\mathrm{A}),(\mathrm{C} 2)$ is not needed taking into account lemma 1.5 and finally, added to $(\mathrm{A})$ or $(\mathrm{C} 1),(\mathrm{C} 3)$ is equivalent to the triviality of $t \Phi$. Besides, in [O.II], Ozaki restricts himself to either the case of a field with a $p$-power conductor, or the semi-simple case with $p$ inert in the ground field, which both imply our hypotheses $(\mathrm{A})$ and $t \Phi=(0)$.

Proof. Assuming hypothesis (A) (which implies (DG) by 2.4 (i)) and $t \Phi=(0)$, we immediately get (i) by 3.9. This implies obviously $\left(B_{\infty}\right)^{\Gamma} \simeq B_{0}$ in (ii). Also, 3.9 gives $\left(\widehat{X_{\infty}^{\text {tor }}}\right)^{\Gamma_{m}} \simeq\left({\widehat{B_{\infty}}}^{\text {tor }}\right)^{\Gamma_{m}}, \forall m \geqslant 0$, hence (iii). As for (iv), our hypotheses imply the cohomological triviality of the $\bar{C}_{n}^{\prime}$ 's by 2.7 , hence $\hat{H}^{i}\left(G_{n, m}, B_{n}\right) \simeq$ $\hat{H}^{i}\left(G_{n, m}, \bar{U}_{n}^{\prime}\right) \simeq \hat{H}^{i-2}\left(G_{n, m}, A_{n}\right)$ by 1.6. Finally, taking inductive limits, we get $H^{1}\left(\Gamma, B_{\infty}\right) \simeq H^{1}\left(\Gamma, U_{\infty}^{\prime}\right)$, which means, by $1.6($ iv $)$, that $\left(B_{\infty}\right)_{\Gamma} \simeq\left(X_{\infty}^{t o r}\right)_{\Gamma}$, and the proof is complete.

In the introduction of section 2, we recalled that $A_{n}$ and $B_{n}$ are not isomorphic in general. But in many particular cases ([KS], [BN1], $[\mathrm{C}],[\mathrm{O} . \mathrm{I}]$, etc...) they 
become asymptotically isomorphic if Greenberg's conjecture holds true. In the same vein:

Corollary 3.11. If $F$ satisfies $(A)$ and $t \Phi=(0)$, and if Greenberg's conjecture holds for the pair $(F, p)$, then $A_{m} \simeq B_{m}, \forall m>>0$ (not canonically).

Proof. Greenberg's conjecture for $(F, p)$ asserts that the $A_{n}$ 's are bounded or, equivalently (because of Sinnott's index formula), the $B_{n}$ 's are bounded. In this case, $\Gamma_{n}=\operatorname{Gal}\left(F_{\infty} / F_{n}\right)$ acts trivially on $A_{n}$ and $B_{n}$ for $n>>0$. It follows that, for $n>>>>0, \hat{H}^{0}\left(G_{n, m}, A_{n}\right) \simeq A_{m}$ and $\hat{H}^{0}\left(G_{n, m}, B_{n}\right) \simeq B_{m}$, hence, by applying 3.10 (iv), $A_{m} \simeq B_{m}$ (not canonically).

Note that the isomorphism $A_{n} \simeq B_{n}$, for $n$ large, does not commute a priori with projective limits, since $\lim _{A_{n}} \simeq X_{\infty}$ while $\lim _{B_{n}}=(0)$ under (DG) and Greenberg's conjecture.

\section{Co-DESCEnT For units MOdUlo CIRCUlar Units}

$F$ still denotes a totally real abelian field. We aim to study co-descent for the module $Y_{\infty}=\lim B_{n}$, i.e. to determine the kernels and cokernels of the natural maps $h_{n}:\left(Y_{\infty}\right)_{\Gamma_{n}} \rightarrow B_{n}$. It is known that the Ker $h_{n}$ 's and Coker $h_{n}$ 's are bounded ([B3], $[\mathrm{N}])$, but we are looking for a more precise description.

Lemma 4.1. Under (DG), for any $n \geqslant 0$, we have a canonical exact sequence:

$$
0 \rightarrow \Psi_{n} \rightarrow \operatorname{Ker} h_{n} \rightarrow \bar{C}_{n} / \widetilde{C}_{n} \rightarrow \bar{U}_{n} / \widetilde{U}_{n} \rightarrow \text { Coker } h_{n} \rightarrow 0
$$

Here $\widetilde{U}_{n}$ denotes the universal norms for units as in 3.5, i.e. the image of the natural map $\left(\bar{U}_{\infty}\right)_{\Gamma_{n}} \rightarrow \bar{U}_{n}$.

Proof. Applying the snake lemma to the commutative diagram:

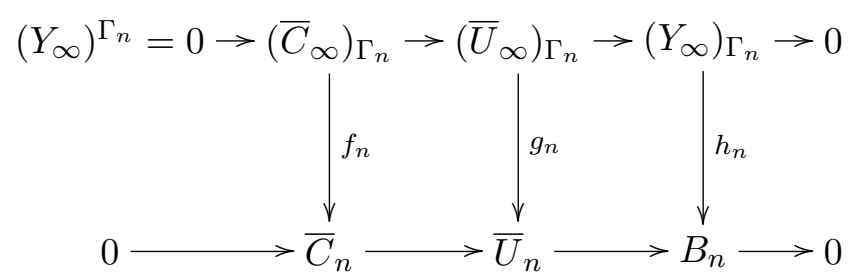

we get an exact sequence (with the notations of 3.4):

$$
\begin{gathered}
0 \rightarrow \text { Ker } f_{n} \simeq\left(Q_{\infty}\right)^{\Gamma_{n}} \rightarrow \text { Ker } g_{n} \simeq\left(R_{\infty}\right)^{\Gamma_{n}} \rightarrow \text { Ker } h_{n} \rightarrow \text { Coker } f_{n} \simeq \bar{C}_{n} / \widetilde{C}_{n} \\
\rightarrow \text { Coker } g_{n} \simeq \bar{U}_{n} / \widetilde{U}_{n} \rightarrow \text { Coker } h_{n} \rightarrow 0 .
\end{gathered}
$$

By the definition of $\Psi_{n}$ (see 3.5), this gives the desired result. 
Our next task is to describe the asymptotical values of the modules appearing in the exact sequence (5). Before going further, we need additional notations about the theory of adjoints. Let $M$ be a finitely generated torsion $\Lambda$-module. For each prime ideal $\mathfrak{p}$ of height one in $\Lambda$, let $M_{\mathfrak{p}}:=M \otimes \Lambda_{\mathfrak{p}}$, where $\Lambda_{\mathfrak{p}}$ is the localization of $\Lambda$ at $\mathfrak{p}$. The cokernel $\beta(M)$ of the canonical map $M \rightarrow \oplus_{\mathfrak{p}} M_{\mathfrak{p}}$ is by definition the co-adjoint of $M$. Let $\alpha(M):=\operatorname{Hom}_{\mathbb{Z}_{p}}\left(\beta(M), \mathbb{Q}_{p} / \mathbb{Z}_{p}\right)$, which we make into a $\Lambda$-module by defining $(\sigma . f)(y):=f\left(\sigma^{-1} . y\right)$ for $\sigma \in \Lambda, y \in \beta(M)$ and $f \in \alpha(M)$. Then $\alpha(M)$ is called the adjoint of $M$. For further details about adjoints, see [Iw2] or [W]. Moreover, for any $\mathbb{Z}_{p}$-module $M$, the Pontryagin dual of $M$ is denoted by $\widehat{M}:=\operatorname{Hom}_{\mathbb{Z}_{p}}\left(M, \mathbb{Q}_{p} / \mathbb{Z}_{p}\right)$.

Lemma 4.2. Under ( $D G)$, taking inductive limits w.r.t. extension maps, we have

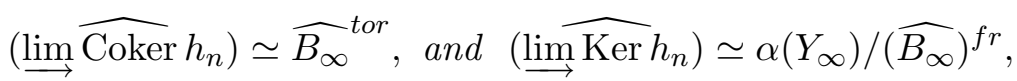

where (.) fr denotes the $\mathbb{Z}_{p}$-torsion free quotient.

Proof. Let us take inductive limits on the tautological exact sequence:

$$
0 \rightarrow \operatorname{Ker} h_{n} \rightarrow\left(Y_{\infty}\right)_{\Gamma_{n}} \rightarrow B_{n} \rightarrow \text { Coker } h_{n} \rightarrow 0 .
$$

For $n \geqslant m$, the transition maps $\left(Y_{\infty}\right)_{\Gamma_{m}} \rightarrow\left(Y_{\infty}\right)_{\Gamma_{n}}$ are $\frac{\gamma^{p^{n}}-1}{\gamma^{p^{m}}-1}$, where $\gamma$ is a topological generator of $\Gamma$. Moreover, the $\left(Y_{\infty}\right)_{\Gamma_{n}}$ 's being finite, $\underline{\lim }\left(Y_{\infty}\right)_{\Gamma_{n}} \simeq$ $\beta\left(Y_{\infty}\right)$ ([Iw2], [W]). By duality, we get an exact sequence:

$$
0 \rightarrow\left(\underline{\lim } \widehat{\operatorname{Coker}} h_{n}\right) \rightarrow \widehat{B_{\infty}} \rightarrow \alpha\left(Y_{\infty}\right) \rightarrow\left(\underline{\lim \operatorname{Ker}} h_{n}\right) \rightarrow 0 .
$$

But an adjoint module has no non zero finite submodule, which shows what we want.

For future use, let us record here a "Spiegelung" result extracted from the proof of lemma 4.2:

Lemma 4.3. Under $(D G)$, suppose moreover that $\lim \operatorname{Ker} h_{n}=(0)$ (as happens e.g. in case $(A)$ or in the semi-simple case). Then $\widehat{Y_{\infty}} \simeq \alpha\left(\widehat{B_{\infty}}\right)$.

Proof. If $\underline{\lim } \operatorname{Ker} h_{n}=(0)$, then ${\widehat{B_{\infty}}}^{f r} \simeq \alpha\left(Y_{\infty}\right)$ by 4.2, which shows in particular that $\widehat{B_{\infty}}$ and $Y_{\infty}$ have the same $\lambda$ and $\mu$-invariants. But $\mu\left(Y_{\infty}\right)=0$ ([FG], [B3] $)$, hence $\alpha\left(\widehat{B_{\infty}}\right) \simeq \operatorname{Hom}\left(\widehat{B_{\infty}}, \mathbb{Z}_{p}\right)([\mathrm{Iw} 2],[\mathrm{W}]) \simeq \operatorname{Hom}\left({\widehat{B_{\infty}}}^{f r}, \mathbb{Z}_{p}\right) \simeq \alpha\left({\widehat{B_{\infty}}}^{f r}\right)$. Applying again the adjoint functor, we get $\alpha\left(\widehat{B_{\infty}}\right) \simeq \alpha\left(\alpha\left(Y_{\infty}\right)\right) \simeq Y_{\infty}$, because $Y_{\infty}$ has no non trivial finite submodule by (DG).

The following does not need hypothesis (DG) : 
Lemma 4.4. For $m>>0$, the natural map $\bar{U}_{m} / \widetilde{U}_{m} \rightarrow \bar{U}_{m}^{\prime} / \widetilde{U}_{m}^{\prime}$ is an isomorphism. In particular, the $\bar{U}_{n} / \widetilde{U}_{n}$ 's stabilize.

Proof. The kernel of the map $\bar{U}_{m} / \widetilde{U}_{m} \rightarrow \bar{U}_{m}^{\prime} / \widetilde{U}_{m}^{\prime}$ is $\bar{U}_{m} \cap \widetilde{U}_{m}^{\prime} / \widetilde{U}_{m}$, which is obviously trivial for $m>>0$. The cokernel is $\bar{U}_{m}^{\prime} / \widetilde{U}_{m}^{\prime} \bar{U}_{m}$; but we have seen in the proof of theorem 1.3 that, for $n \geqslant m>>0$, the norm map $\bar{U}_{n}^{\prime} / \bar{U}_{n} \rightarrow \bar{U}_{m}^{\prime} / \bar{U}_{m}$ is surjective, hence the cokernel in question is null. The stabilization of the $\bar{U}_{n}^{\prime} / \widetilde{U}_{n}^{\prime}$ 's has been shown by Kuz'min (see [Ku], proof of proposition 8.2).

We can now show the main result of this section on co-descent:

Theorem 4.5. Under $(D G)$, the Coker $h_{n}$ 's and Ker $h_{n}$ 's stabilize w.r.t. extension maps. For $n>>0$, Coker $h_{n} \simeq \widehat{\left(\widehat{B}_{\infty}^{\text {tor }}\right)} \simeq B_{\infty} / \operatorname{Div}\left(B_{\infty}\right)$, and Ker $h_{n}$ takes place in an exact sequence:

$$
0 \rightarrow \Psi \rightarrow \operatorname{Ker} h_{n} \rightarrow t \Phi \stackrel{\beta}{\rightarrow} X_{\infty}^{\prime} \text { tor } \stackrel{\epsilon}{\rightarrow} \widehat{\left(\widehat{B}_{\infty}^{\text {tor }}\right)} .
$$

Proof. For $n>>0$, the exact sequence (5) in 4.1 becomes :

$$
0 \rightarrow \Psi \rightarrow \operatorname{Ker} h_{n} \rightarrow \Phi \rightarrow \bar{U}_{n} / \widetilde{U}_{n} \rightarrow \text { Coker } h_{n} \rightarrow 0 .
$$

By 4.4 , the $\bar{U}_{n} / \widetilde{U}_{n}$ 's stabilize, hence the Ker $h_{n}$ 's and Coker $h_{n}$ 's also. The isomorphism Coker $h_{n} \simeq \widehat{\left(\widehat{B}_{\infty}^{\text {tor }}\right)}$ for $n>>0$ is then just a restatement of lemma 4.2. Taking $\mathbb{Z}_{p}$-torsion in the exact sequence (6), we get the desired result for $\operatorname{Ker} h_{n}$.

It would be desirable to compare the two exact sequences in 3.7 and 4.5 but a priori, we can't see any natural map between Coker $j_{n}$ and Ker $h_{n}$, except in particular cases:

Corollary 4.6. Under $(D G)$, and for $n>>0$ :

a) $\operatorname{Ker} h_{n}=(0)$ if and only if Coker $j_{n}=(0)$.

b) If $\Psi=(0)$, then $\operatorname{Ker} h_{n} \simeq$ Coker $j_{n}$.

Proof. a) clearly follows from b). To prove b), we must show that the two maps $\alpha, \beta: t \Phi \rightarrow X_{\infty}^{\prime t o r}$ in 3.7 and 4.5 coincide up to isomorphism. While $\beta$ is just induced by the natural map $\bar{C}_{n} / \widetilde{C}_{n} \rightarrow \bar{U}_{n}^{\prime} / \widetilde{U}_{n}^{\prime}$, we must trace $\alpha$ back to the proof of theorem 2.3 and lemma 3.6: for $n \geqslant m>>0$, the cohomological map $H^{1}\left(G_{n, m}, \bar{C}_{n}\right) \rightarrow H^{1}\left(G_{n, m}, \bar{U}_{n}^{\prime}\right)$ induces a map $H^{1}\left(G_{n, m}, \bar{C}_{n} / \widetilde{C}_{n}\right)=$ $\operatorname{Hom}\left(G_{n, m}, \bar{C}_{n} / \widetilde{C}_{n}\right) \rightarrow H^{1}\left(G_{n, m}, \bar{U}_{n}^{\prime} / \widetilde{U}_{n}^{\prime}\right)=\operatorname{Hom}\left(G_{n, m}, \bar{U}_{n}^{\prime} / \widetilde{U}_{n}^{\prime}\right)$; after identifying the first Hom group (resp. the second) with $p^{n-m} t \Phi$ (resp. $p^{n-m} X_{\infty}^{\prime}{ }^{t o r}$ ), we recover $\alpha$. 


\section{Remarks:}

1) Using $\beta$, we can easily give a description of the cokernel of the map $\epsilon$ : $X_{\infty}^{\prime}{ }^{\text {tor }} \rightarrow\left({\widehat{\bar{B}_{\infty}}}^{\text {tor }}\right)$ in theorems 3.7 and 4.5. Writing $(\cdot)^{f r}$ for the quotient modulo $\mathbb{Z}_{p}$-torsion, the exact sequence (6) in the proof of 4.5 immediately shows (under (DG) and for $n>>0$ ) that Coker $\epsilon$ is just the cokernel of the natural $\operatorname{map}(\Phi)^{f r} \rightarrow\left(\bar{U}_{n}^{\prime} / \widetilde{U}_{n}^{\prime}\right)^{f r}$ (which is injective). Note that, according to Kuz'min's results, $\left(\bar{U}_{n}^{\prime} / \widetilde{U}_{n}^{\prime}\right)^{f r}$ is no other than $\bar{U}_{n}^{\prime} / \widehat{U}_{n}^{\prime}$.

2) Assuming Greenberg's conjecture, we can conveniently compute the order of Coker $\epsilon$. Under (DG), Greenberg's conjecture is equivalent to the triviality of $Y_{\infty}$, hence Ker $h_{n}=(0)$ and $B_{n} \simeq$ Coker $h_{n}$ for all $n \geqslant 0$, and the exact sequence in theorem 4.5 becomes, for all $n>>0$ :

$$
0 \rightarrow t \Phi \rightarrow A_{n}^{\prime} \rightarrow B_{n} \rightarrow \text { Coker } \epsilon \rightarrow 0 .
$$

This yields a $p$-adic equivalence of orders: $\mid$ Coker $\epsilon\left|\stackrel{p}{\sim} \frac{\left|B_{n}\right|}{\left|A_{n}\right|} \cdot\right| D_{n}|\cdot| t \Phi \mid$. Let us denote by $c_{\infty}$ the asymptotic value of the $p$-parts of the Sinnott constants $c\left(F_{n}\right)$. Then: $\mid$ Coker $\epsilon|\stackrel{p}{\sim}| c_{\infty}|\cdot| D_{\infty}|\cdot| t \Phi \mid$. Thus we cannot expect Coker $\epsilon$ to vanish in general.

Without Greenberg's conjecture, the ambiguities in theorem 4.5 can be removed in the special case $(\mathrm{A})$ :

Proposition 4.7. Under (A), for any $n \geqslant 0$, we have an exact sequence:

$$
0 \rightarrow \operatorname{Ker} h_{n} \rightarrow t \Phi \rightarrow\left(X_{\infty}^{\text {tor }}\right)^{\Gamma_{n}} \rightarrow \text { Coker } h_{n} \rightarrow 0 .
$$

If moreover $t \Phi=(0)$, co-descent is summarized in an exact sequence:

$$
0 \rightarrow\left(Y_{\infty}\right)_{\Gamma_{n}} \rightarrow B_{n} \rightarrow\left(X_{\infty}^{\text {tor }}\right)^{\Gamma_{n}} \simeq\left(\widehat{\bar{B}_{\infty}^{\text {tor }}}\right)_{\Gamma_{n}} \rightarrow 0
$$

(to be compared with [O.II], theorem 1, and [BN1], theorem 3.5).

Proof. Under (A), knowing that $B_{n} \simeq \bar{U}_{n}^{\prime} / \bar{C}_{n}^{\prime}$ (lemma 2.7), the same argument as in lemma 4.1 gives an exact sequence:

$$
0 \rightarrow \operatorname{Ker} h_{n} \rightarrow \bar{C}_{n}^{\prime} / \widetilde{C}_{n}^{\prime} \rightarrow \bar{U}_{n}^{\prime} / \widetilde{U}_{n}^{\prime} \rightarrow \text { Coker } h_{n} \rightarrow 0 .
$$

But the $p$-adic valuation gives rise to $0 \rightarrow \bar{C}_{n} \rightarrow \bar{C}_{n}^{\prime} \rightarrow \mathbb{Z}_{p} \rightarrow 0$, from which it follows easily that $\bar{C}_{n} / \widetilde{C}_{n} \simeq \bar{C}_{n}^{\prime} / \widetilde{C}_{n}^{\prime}$. Besides, because $s=1, \bar{C}_{n} / \widetilde{C}_{n} \simeq t \Phi$ and $\bar{U}_{n}^{\prime} / \widetilde{U}_{n}^{\prime} \simeq\left(X_{\infty}^{t o r}\right)^{\Gamma_{n}}$. Finally, if $t \Phi=(0)$, then $\left(X_{\infty}^{t o r}\right)^{\Gamma_{n}} \simeq$ Coker $h_{n}$, which implies that Coker $\epsilon=(0)$, whence the exact sequence $(7)$.

The co-descent exact sequence (7) yields : 
Another proof of corollary 3.11. Under (DG), Greenberg's conjecture asserts the nullity of $Y_{\infty}$. If $F$ satisfies $(\mathrm{A})$ and $t \Phi=(0)$, theorem 3.4 then gives an isomorphism $B_{n} \simeq\left(X_{\infty}\right)^{\Gamma_{n}}$ for all $n \geqslant 0$. But in case (A), $A_{n} \simeq\left(X_{\infty}\right)_{\Gamma_{n}}$ for all $n \geqslant 0$, hence $A_{n} \simeq B_{n} \simeq X_{\infty}$ for all $n>>0$, modulo Greenberg's conjecture.

\section{Descent and CO-Descent in the Semi-Simple Case}

We now intend to do a characterwise study in the semi-simple case, i.e. when $F$ is a totally real abelian field such that $p \nmid[F: \mathbb{Q}]$. Let us remark right away that semi-simplicity implies hypothesis (DG) (proposition 2.4), hypothesis (R) and the triviality of $t \Phi$ (corollary 2.6), so that the exact sequences in theorems 3.7 and 4.5 break down to give, for any $n$ large enough, $\Psi \simeq$ Coker $j_{n} \simeq \operatorname{Ker} h_{n}$ and

$$
0 \rightarrow X_{\infty}^{\prime \text { tor }} \stackrel{\epsilon}{\rightarrow}\left({\widehat{\bar{B}_{\infty}^{\text {tor }}}}^{\mathrm{a}}\right) \rightarrow \text { Coker } \epsilon \rightarrow 0
$$

Let $\Delta:=\operatorname{Gal}(F / \mathbb{Q})$ and $\hat{\Delta}:=\operatorname{Hom}\left(\Delta, \overline{\mathbb{Q}}_{p}^{\times}\right)$. If $M$ is a $\mathbb{Z}_{p}[\Delta]$-module and $\chi \in \hat{\Delta}$ is a character of $\Delta$, the $\chi$-part of $M$ is denoted by $M(\chi)$. Recall that $M(\chi)=e_{\chi} M$, with

$$
e_{\chi}:=\frac{1}{|\Delta|} \sum_{\delta \in \Delta} \operatorname{Tr}(\chi(\delta)) \delta^{-1} \in \mathbb{Z}_{p}[\Delta] .
$$

Let $R_{\chi}:=\mathbb{Z}_{p}[\Delta](\chi)$ and $\Lambda_{\chi}:=R_{\chi}[[\Gamma]]$.

We'll have to distinguish between the non-split case $(\chi(p) \neq 1)$ and the split case $(\chi(p)=1)$. In the non-split case we can get full, non asymptotic results, just as in case $(\mathrm{A})$ :

Theorem 5.1. For any character $\chi \in \hat{\Delta}$ such that $\chi(p) \neq 1$ :

(i) The $B_{n}(\chi)^{\prime}$ 's satisfy Galois descent, i.e. $\left(B_{n}(\chi)\right)^{G_{n, m}} \simeq B_{m}(\chi), \forall n \geqslant m \geqslant$ 0 (cp. 3.10).

(ii) For any $n \geqslant 0$, we have an exact sequence of co-descent:

$$
0 \rightarrow\left(Y_{\infty}\right)_{\Gamma_{n}}(\chi) \rightarrow B_{n}(\chi) \rightarrow\left(X_{\infty}^{\text {tor }}\right)^{\Gamma_{n}}(\chi) \simeq\left({\widehat{\left(\bar{B}^{\text {tor }}\right.}}_{\Gamma_{n}}\right)(\chi) \rightarrow 0
$$

(cp. 4.7).

Let us first determine separately the $\chi$-parts $\Psi(\chi)$ and $($ Coker $\epsilon)(\chi)$ :

Lemma 5.2. For any character $\chi \in \hat{\Delta}$ such that $\chi(p) \neq 1$, we have $\Psi_{n}(\chi)=(0)$ for any $n \geqslant 0$. 
Proof. By definition (see 3.5), $\Psi_{n}$ is a quotient of $\mathbb{Z}_{p}\left[S_{n}\right]$, where $S_{n}$ is the set of $p$ primes of $F_{n}$. By the semi-simplicity hypothesis, $\Delta$ acts naturally on any $\mathbb{Z}_{p}\left[S_{n}\right]$, and this action can be read on $\mathbb{Z}_{p}\left[S_{0}\right]$ alone thanks to hypothesis $(\mathrm{R})$. Denoting by $\nabla$ the decomposition subgroup of $\Delta$ relative to any chosen $p$-prime in $S_{0}$, we have obviously $\mathbb{Z}_{p}\left[S_{0}\right] \simeq \mathbb{Z}_{p}[\Delta / \nabla]$ as $\Delta$-modules, so that $\mathbb{Z}_{p}\left[S_{0}\right](\chi) \simeq R_{\chi}[\Delta / \nabla]$. Moreover, $\chi(p)=1$ if and only if $\chi$ factors through $\Delta / \nabla$, hence $\mathbb{Z}_{p}\left[S_{0}\right](\chi)=(0)$ if $\chi(p) \neq 1$. Note that if $\chi$ is a non trivial character such that $\chi(p)=1$, this argument shows that $\mathbb{Z}_{p}\left[S_{0}\right](\chi) \simeq R_{\chi}$.

Lemma 5.3. For any character $\chi \in \hat{\Delta}$ such that $\chi(p) \neq 1$, we have

$$
(\text { Coker } \epsilon)(\chi)=(0) \text {. }
$$

Proof. We have seen in remark 1) after 4.6 that, for $n$ large enough, Coker $\epsilon$ is a subquotient of $\underset{v \mid p}{\tilde{\oplus}} \overline{F_{n, v}^{\times}} / \hat{F}_{n, v}^{\times}$. But this sum $\tilde{\oplus}$ is in turn a submodule of $\mathbb{Z}_{p}\left[S_{n}\right]$, and the same argument as in lemma 5.2 applies.

Proof of theorem 5.1. To show 5.1 (i), we cannot apply directly theorem 3.7, whose statement is asymptotic. However, for any $n \geqslant 0$, we have seen in lemma 3.3 that Coker $j_{n} \simeq \operatorname{Ker}\left(H^{1}\left(\Gamma_{n}, C_{\infty}\right) \rightarrow H^{1}\left(\Gamma_{n}, U_{\infty}\right)\right)$ and in the proof of lemma 3.6 that $\Psi_{n} \simeq \operatorname{Ker}\left(\operatorname{Div} H^{1}\left(\Gamma_{n}, C_{\infty}\right) \rightarrow H^{2}\left(\Gamma_{n}, R_{n}\right)\right)$. Since $t \Phi=(0)$ here, Div $H^{1}\left(\Gamma_{n}\right.$, $\left.C_{\infty}\right)=H^{1}\left(\Gamma_{n}, C_{\infty}\right)$. Besides, $D_{n}(\chi)=(0)$ as a quotient of $\mathbb{Z}_{p}\left[S_{n}\right](\chi)$, hence the end of the proof of 1.3 (ii) applies to yield $H^{2}\left(\Gamma_{n}, R_{n}\right)(\chi) \simeq \operatorname{Div} H^{1}\left(\Gamma_{n}, U_{\infty}\right)(\chi)$. We conclude that $\left(\right.$ Coker $\left.j_{n}\right)(\chi) \simeq \Psi_{n}(\chi)=(0)$ by lemma 5.2.

Since $t \Phi=(0)$, the injectivity in 5.1 (ii) follows obviously from lemma 5.2 and the exact sequence (5) in lemma 4.1. The same exact sequence and lemma 5.3 show that $\left(\bar{U}_{n} / \widetilde{U}_{n}\right)^{\text {tor }}(\chi) \simeq\left(\right.$ Coker $\left.h_{n}\right)(\chi)$. But again, since $D_{n}(\chi)=(0)$, we can apply the argument in lemma 4.4 to show that $\left(\bar{U}_{n} / \widetilde{U}_{n}\right)(\chi) \simeq\left(\bar{U}_{n}^{\prime} / \widetilde{U}_{n}^{\prime}\right)(\chi)$, whence $\left(\bar{U}_{n} / \widetilde{U}_{n}\right)^{\text {tor }}(\chi) \simeq\left(X_{\infty}^{\prime \text { tor }}\right)^{\Gamma_{n}}(\chi)=\left(X_{\infty}^{\text {tor }}\right)^{\Gamma_{n}}(\chi)$. Besides, lemma 5.3 means that $\left(X_{\infty}^{\prime}{ }^{\text {tor }}\right)(\chi) \simeq\left(\widehat{{\widehat{B_{\infty}}}^{\text {tor }}}\right)(\chi)$.

Corollary 5.4 (cp. 3.11). Suppose moreover that Greenberg's conjecture is valid. Then $A_{n}(\chi) \simeq B_{n}(\chi)$ for all $n$ large enough.

Proof. By 5.1 (ii) and the same argument as after 4.7, we immediately get $A_{n}^{\prime}(\chi) \simeq$ $B_{n}(\chi)$. But we have seen that $D_{n}(\chi)=(0)$.

The split case $(\chi(p)=1)$ is notoriously more complicated because, when performing descent or co-descent, we could run into trivial zeroes of $p$-adic $L$-functions (on the analytic side). The argument in lemma 5.2 only tells us that $\Psi(\chi)$ and $($ Coker $\epsilon)(\chi)$ are cyclic over $R_{\chi}$ if $\chi \neq 1$. For further partial results, we refer to 
[N] (whose primary goal, however, is different from here).

We'll conclude by using descent and co-descent to gather information on the parenthood between $A_{n}$ and $B_{n}$ (or $X_{\infty}$ and $Y_{\infty}$ ) without assuming Greenberg's conjecture.

Proposition 5.5 (cp. [O.II], theorem 2). For any character $\chi \in \hat{\Delta}$ such that $\chi(p) \neq 1$ and $A_{\infty}^{\Gamma}(\chi)$ is cyclic, we have an isomorphism (non canonical) of $\Lambda_{\chi^{-}}$ modules $Y_{\infty}(\chi) \simeq X_{\infty}^{f r}(\chi)$, where $X_{\infty}^{f r}$ is the $\mathbb{Z}_{p}$-torsion free quotient $X_{\infty} / X_{\infty}^{\text {tor }}$.

Proof. It is well known that $\bar{U}_{0}\left(\chi^{-1}\right) \simeq \mathbb{Z}_{p}\left[\chi^{-1}\right]$ if $\chi$ is not the trivial character, and trivial otherwise. But $\left(\widehat{B_{\infty}}(\chi)\right)_{\Gamma}$ is the dual of $B_{\infty}\left(\chi^{-1}\right)^{\Gamma} \simeq B_{0}\left(\chi^{-1}\right)$ by 5.1 (i). As $B_{0}\left(\chi^{-1}\right)$ is a quotient of $\bar{U}_{0}\left(\chi^{-1}\right)$, Nakayama's lemma shows that $\widehat{B_{\infty}}(\chi)$ is cyclic over $\Lambda_{\chi}$, and so is $\alpha\left(Y_{\infty}\right)(\chi)$, which is a quotient of $\widehat{B_{\infty}}(\chi)$ since $\left(\right.$ Ker $\left.h_{n}\right)(\chi)=(0)$ (see the proof of 4.3). As $\mu\left(Y_{\infty}\right)=0$, the same argument as in 4.7 shows that $Y_{\infty}(\chi) \simeq \alpha\left(\alpha\left(Y_{\infty}\right)(\chi)\right) \simeq \operatorname{Hom}\left(\alpha\left(Y_{\infty}\right)(\chi), \mathbb{Z}_{p}\right)$ is also cyclic over $\Lambda_{\chi}$, and so it is of the form $Y_{\infty}(\chi) \simeq \Lambda_{\chi} /\left(f_{\chi}(T)\right)$, for the projective dimension of $Y_{\infty}(\chi)$ over $\Lambda_{\chi}$ is less than 1 (here we have identified in a classical way $\Lambda_{\chi}$ and the ring $R_{\chi}[[T]]$ of formal power series).

Moreover the tautological exact sequence

$$
0 \rightarrow X_{\infty}^{t o r}(\chi) \rightarrow X_{\infty}(\chi) \rightarrow X_{\infty}^{f r}(\chi) \rightarrow 0
$$

gives by co-descent

$$
0 \rightarrow X_{\infty}^{t o r}(\chi)_{\Gamma} \rightarrow A_{0}(\chi) \simeq X_{\infty}(\chi)_{\Gamma} \rightarrow X_{\infty}^{f r}(\chi)_{\Gamma} \rightarrow 0
$$

which means, by 1.6 (iii), that $A_{\infty}^{\Gamma}(\chi) \simeq X_{\infty}^{f r}(\chi)_{\Gamma}$. Thus the hypothesis that $A_{\infty}^{\Gamma}(\chi)$ is cyclic is equivalent to the cyclicity of $X_{\infty}^{f r}(\chi)$ over $\Lambda_{\chi}$. In this case, $X_{\infty}^{f r}(\chi) \simeq \Lambda_{\chi} /\left(f_{\chi}(T)\right)$ since we know by the Main Conjecture that $X_{\infty}(\chi)$ and $Y_{\infty}(\chi)$ have the same characteristic series. Hence $Y_{\infty}(\chi) \simeq X_{\infty}^{f r}(\chi)$.

Corollary 5.6. Keeping the hypotheses of 5.5, for all $n \geqslant 0$, we have an exact sequence

$$
0 \rightarrow\left(X_{\infty}^{\text {tor }}\right)_{\Gamma_{n}}(\chi) \rightarrow A_{n}(\chi) \rightarrow B_{n}(\chi) \rightarrow\left(X_{\infty}^{\text {tor }}\right)^{\Gamma_{n}}(\chi) \rightarrow 0 .
$$

Proof. The snake lemma applied to the exact sequence

$$
0 \rightarrow X_{\infty}^{\text {tor }}(\chi) \rightarrow X_{\infty}(\chi) \rightarrow X_{\infty}^{f r}(\chi) \simeq Y_{\infty}(\chi) \rightarrow 0
$$

gives

$$
\left(X_{\infty}^{f r}\right)^{\Gamma_{n}}(\chi)=0 \rightarrow\left(X_{\infty}^{t o r}\right)_{\Gamma_{n}}(\chi) \simeq A_{n}(\chi) \rightarrow\left(Y_{\infty}\right)_{\Gamma_{n}}(\chi) \rightarrow 0
$$

It then suffices to apply the exact sequence (8) in 5.1.

Note that Greenberg's conjecture implies the nullity of the above map $A_{n}(\chi) \rightarrow$ $B_{n}(\chi)$ for $n$ large, although $A_{n}(\chi) \simeq B_{n}(\chi)(\operatorname{cf} 5.4)$. 


\section{Appendix : about the $\Lambda$-Freeness of $\bar{C}_{\infty}$ \\ BY J.-R. BELLIARD}

Let $F$ be an abelian totally real number field (without any further restriction). In the paper [B1] we gave a sufficient condition to $\Lambda$-freeness of the $\Lambda$-module $\bar{C}_{\infty}$ associated to the cyclotomic $\mathbb{Z}_{p}$-extension $F_{\infty} / F$. There, amongst other hypotheses, we assumed that $p$ was unramified in the base field (denoted $K$ there and $F$ here). Now, due to the applications given in the main part of this paper, it seems worthwhile to write down precisely the relevant condition without assuming anything about ramification at $p$.

Lemma 6.1. Let $L \subset F_{\infty}$ be the maximal subfield of $F_{\infty}$ such that $p$ is (at most) tamely ramified in $L$ (under hypothesis $(R), L$ is the field $F^{\text {tam }}$ introduced after 2.3). Put $\Lambda_{L}:=\mathbb{Z}_{p}\left[\left[\operatorname{Gal}\left(L_{\infty} / L\right)\right]\right]$, where $L_{\infty} / L$ is the cyclotomic $\mathbb{Z}_{p}$-extension of L. Then we have:

(1) As a group, $\bar{C}_{\infty}$ only depends on $F_{\infty}$ not on $F$.

(2) $L_{\infty}=F_{\infty}$.

(3) $\bar{C}_{\infty}$ is $\Lambda$-free if and only if $\bar{C}_{\infty}$ is $\Lambda_{L}$-free.

Proof. 1 is clear. 2 is lemma 1.2 of [B3]. By theorem 2.2 of [B2], the $\Lambda$-freeness is equivalent to an asymptotic condition (namely the "Asymptotic Galois Descent" property for the $\bar{C}_{n}$ 's). But there exists some $N \in \mathbb{N}$ and some $b \in \mathbb{Z}$ such that for all $n \geq N$ we have $F_{n}=L_{n+b}$ : the equivalence in 3 follows.

In the light of lemma 6.1, as far as the $\Lambda$-freeness of $\bar{C}_{\infty}$ is concerned, we could (but shall not in the sequel) assume without loss of generality that $p$ is (at most) tamely ramified in $F$. Then we might just refer to [B1] for all proofs. But we thought it would be better to give them here, including most of the details, taking this opportunity to simplify the arguments of [B1] by translating them at infinite level.

We need a few more notations. They are close to those used in [B1] but formally not exactly the same. Let $\mathcal{P}$ be the set of rational primes $l \neq p$ which are ramified in $F / \mathbb{Q}$. The case $\mathcal{P}=\emptyset$ is obvious but allowed. For all supernatural numbers $t$ we put $\mathbb{Q}(t)=\mathbb{Q}\left(\zeta_{t}\right)$. For all $J \subset \mathcal{P}$ and all $n \in \mathbb{N} \cup\{\infty\}$, we put $F_{n}(J):=\mathbb{Q}\left(\prod_{l \in J} l^{\infty} p^{\infty}\right) \cap F_{n}$. We shall abbreviate by $F(J):=F_{0}(J), G(J)=$ $\operatorname{Gal}\left(F_{\infty}(J) / \mathbb{B}_{\infty}\right)$, where $\mathbb{B}_{\infty}$ is the cyclotomic $\mathbb{Z}_{p}$-extension of $\mathbb{Q}$, and $G=G(\mathcal{P})$. For any abelian number field $K$ we write $\operatorname{cond}(K)$ for its conductor, that is the minimal $n \in \mathbb{N}$ such that $K \subset \mathbb{Q}(n)$. For all $n \in \mathbb{N}$ and all $J \subset \mathcal{P}$ we shall denote by $\varepsilon_{n}(J)$ the following cyclotomic number (unit if $J \neq \emptyset$ ) :

$$
\varepsilon_{n}(J):=N_{\mathbb{Q}\left(\operatorname{cond}\left(F_{n}(J)\right)\right) / F_{n}(J)}\left(1-\zeta_{\operatorname{cond}\left(F_{n}(J)\right)}\right) .
$$


It follows from the classical distribution relations that, for $n \geq 1$, the $\varepsilon_{n}(J)$ 's form a norm coherent sequence. Therefore, fixing a generator $\gamma$ of $\operatorname{Gal}\left(\mathbb{B}_{\infty} / \mathbb{Q}\right)$ we may define $\varepsilon_{\infty}(J) \in \bar{C}_{\infty}$ by the following formula :

$$
\varepsilon_{\infty}(J):=\left\{\begin{array}{l}
\left(\varepsilon_{n}(J)\right)_{n \geq 1} \text { if } J \neq \emptyset \\
\left(\varepsilon_{n}(\emptyset)^{(\gamma-1)}\right)_{n \in \mathbb{N}} \text { else. }
\end{array}\right.
$$

We shall examine the $\Lambda_{L}[G]$-module structure of $\bar{C}_{\infty}$ (recall that $G=G(\mathcal{P})=$ $\left.\operatorname{Gal}\left(F_{\infty} / \mathbb{B}_{\infty}\right)\right)$, and for that it is more convenient to write additively the multiplication in $\bar{C}_{\infty}$ and to keep multiplication for the action of $\Lambda[G]$. Consistently for $\mathbb{B}_{\infty} \subset N \subset K \subset F_{\infty}$ we denote by $T_{K / N}$ the trace (actually the algebraic norm map) acting on elements $x \in \bar{C}_{\infty}^{\operatorname{Gal}\left(F_{\infty} / K\right)}$. The elements $\varepsilon_{\infty}(J)$ are subject to the following distribution relations :

Lemma 6.2. Let $\left(\frac{\mathfrak{A}}{N / K}\right)$ be the (global) Artin symbol for any abelian extension $N / K$ and (fractional) ideal $\mathfrak{A}$ of $K$. For all $J \subset \mathcal{P}$ and all $I \subset J$, put $\mathcal{P}_{I, J}$ for the set of rational primes $l$ dividing $\operatorname{cond}\left(F_{\infty}(J)\right)$ but not dividing $\operatorname{cond}\left(F_{\infty}(I)\right)$. We have :

$$
T_{F_{\infty}(J) / F_{\infty}(I)}\left(\varepsilon_{\infty}(J)\right)=\left(\prod_{l \in \mathcal{P}_{I, J}}\left(1-\left(\frac{(l)}{F_{\infty}(I) / \mathbb{Q}}\right)^{-1}\right)\right) \varepsilon_{\infty}(I) .
$$

Proof. The analogous relations at finite levels are well known. We then just take inverse limits.

Lemma 6.3. The system $\left\{\varepsilon_{\infty}(J), J \subset \mathcal{P}\right\}$ generates $\bar{C}_{\infty}$ (as a module over $\left.\Lambda_{L}\left[\operatorname{Gal}\left(F_{\infty} / \mathbb{B}_{\infty}\right)\right]\right)$.

Proof. This follows from distribution relations (e.g. lemma 6.2) and lemma 2.3 of [Gt].

We now state the ad hoc hypothesis that will ensure the freeness of $\bar{C}_{\infty}$. This "generalized hypothesis (B)" is an immediate generalization of (HB) in [B1].

Definition 6.4. For all $J \subset \mathcal{P}$, we call the norm ideal of $J$ and denote by $N(J)$ the ideal of $\mathbb{Z}_{p}[G(J)]$ generated by traces

$$
N(J):=\left\langle T_{F_{\infty}(J) / F_{\infty}(J-\{l\})} ; l \in J\right\rangle .
$$

We say that the pair $(F, p)$ satisfies the "generalized Hypothesis B" ((gHB) for short) if and only if for all $J \subset \mathcal{P}$ the quotient $\mathbb{Z}_{p}[G(J)] / N(J)$ is torsion free.

This hypothesis is quite technical, but very natural regarding the proof of theorem 6.6. The following proposition shows that (gHB) holds true in many 
usual cases. Indeed all known cases of freedom of $\bar{C}_{\infty}$ are consequences of (gHB) via theorem 6.6.

Proposition 6.5. For all $l \in \mathcal{P}$ let us denote by $I_{l} \subset G$ the inertia subgroup for $l$ in $F_{\infty} / \mathbb{B}_{\infty}$. Recall that for any finite abelian group $H$, we write $\bar{H}$ for its $p$ Sylow subgroup (considered also as the maximal p-quotient of $H$ ). The pair $(F, p)$ satisfies $(g H B)$ as soon as one of the following properties holds :

(1) The $\bar{I}_{l}, l \in \mathcal{P}$ are mutually direct summands. In other words the natural map $\bigoplus_{l \in \mathcal{P}} \bar{I}_{l} \longrightarrow \bar{G}$ is injective.

(2) $\operatorname{dim}_{\mathbb{F}_{p}}(G / p G) \leq 1$, or equivalently $\bar{G}$ is cyclic.

(3) $\# \mathcal{P} \leq 2$

Proof. These sufficient conditions are the exact analogues of those in section IV.1 of [B1]. Note that they concern only the maximal $p$-quotient of the Galois groups involved. Since the (unique) prime above $p$ of $\mathbb{B}_{\infty}$ is tamely ramified in $F_{\infty} / \mathbb{B}_{\infty}$, everything goes as if $p$ were unramified in the base field $F$. Taking this into account, we can repeat verbatim the proofs in [B1].

Theorem 6.6. Let us denote by $r(I)$ the $\mathbb{Z}_{p}$-rank of $\mathbb{Z}_{p}[G(I)] / N(I)$. Assume that $(F, p)$ satisfies $(g H B)$. Fix any total order $\leq$ on the set of all subsets of $\mathcal{P}$ extending the inclusion. Then :

(1) $\forall J \subset \mathcal{P}$ we have isomorphisms of $\Lambda_{L}[G]$-modules :

$$
\begin{aligned}
\frac{\mathbb{Z}_{p}[G(J)]}{N(J)} \otimes_{\mathbb{Z}_{p}} \Lambda_{L} & \cong \frac{\left\langle\varepsilon_{\infty}(J)\right\rangle}{\left\langle\varepsilon_{\infty}(I) ; I \subset J, I \neq J\right\rangle \bigcap\left\langle\varepsilon_{\infty}(J)\right\rangle} \\
& \cong \frac{\left\langle\varepsilon_{\infty}(J)\right\rangle}{\left\langle\varepsilon_{\infty}(I) ; I \leq J, I \neq J\right\rangle \bigcap\left\langle\varepsilon_{\infty}(J)\right\rangle} ;
\end{aligned}
$$

hence all three $\Lambda_{L}[G]$-modules are free over $\Lambda_{L}$ (of ranks $r(J)$ ).

(2) $\bar{C}_{\infty} \cong \bigoplus_{I \subset \mathcal{P}} \Lambda_{L}^{r(I)}$ as $\Lambda_{L}$-modules; in particular $\bar{C}_{\infty}$ is $\Lambda_{L}$-free of rank $[L: \mathbb{Q}]$ and $\Lambda$-free of rank $[F: \mathbb{Q}]$.

Proof. $\bar{C}_{\infty}$ has the same $\Lambda$-rank (resp. $\Lambda_{L}$-rank) as $\bar{U}_{\infty}$, namely $[F: \mathbb{Q}]$ (resp. $\left.[L: \mathbb{Q}]=\left[F_{\infty}: \mathbb{B}_{\infty}\right]\right)$ : see e.g. $[\mathrm{Ku}],[\mathrm{B} 2] \ldots$ By $(\mathrm{gHB})$ the modules $\frac{\mathbb{Z}_{p}[G(J)]}{N(J)} \otimes_{\mathbb{Z}_{p}} \Lambda_{L}$ are $\Lambda_{L}$-free. We first prove that 1 implies 2. From the tautological (split over $\Lambda_{L}$ under (gHB) by 1) exact sequences :

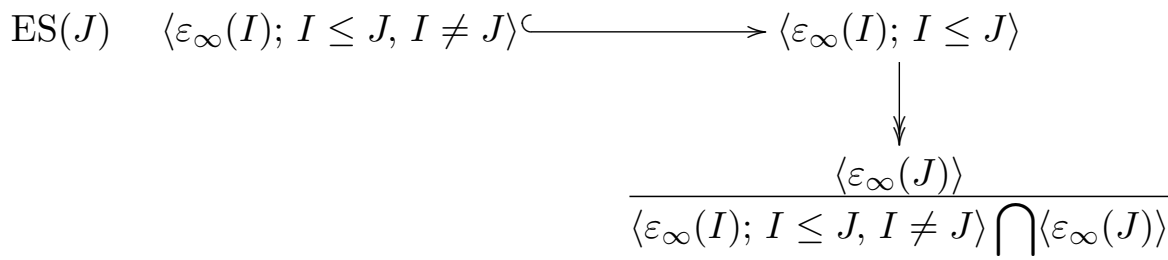


we see that 1 implies 2 by induction. Let us prove 1 .

By lemma 6.2 and by the inclusion

$$
\left\langle\varepsilon_{\infty}(I) ; I \subset J, I \neq J\right\rangle \subset\left\langle\varepsilon_{\infty}(I) ; I \leq J, I \neq J\right\rangle
$$

we have surjective morphisms :

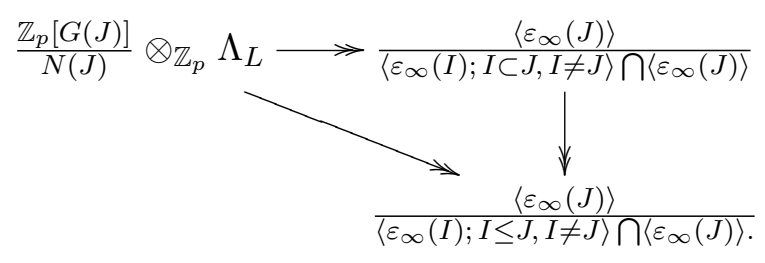

Therefore the required isomorphisms will follow from equalities of $\Lambda_{L}$-ranks. Now :

Lemma 6.7. Recall that for all $I, r(I)$ is the $\mathbb{Z}_{p}$-rank of $\mathbb{Z}_{p}[G(I)] / N(I)$. Put $d(I)=\left[F_{\infty}(I): \mathbb{B}_{\infty}\right]$.

(1) For all $J \subset \mathcal{P}$ we have

$$
r(J)=\sum_{I \subset J}(-1)^{\# J-\# I} d(I)
$$

$$
[L: \mathbb{Q}]=\left[F_{\infty}: \mathbb{B}_{\infty}\right]=d(\mathcal{P})=\sum_{J \subset \mathcal{P}} r(J)
$$

Proof. 1 is proven using character theory and following the same argument as in the proof of proposition 2.11 of [B1] : just replace the function $f_{n}(I)$ there by $d(I)$ here. 2 follows from 1 and the combinatorial lemma 2.14 of [B1]. Details are left to the reader.

We resume the proof of theorem 6.6. For any $\Lambda_{L}$-module $M$ let us denote its $\Lambda_{L}$-rank by $\operatorname{rank}_{\Lambda_{L}}(M)$. Put

$$
t(J):=\operatorname{rank}_{\Lambda_{L}}\left(\frac{\left\langle\varepsilon_{\infty}(J)\right\rangle}{\left\langle\varepsilon_{\infty}(I) ; I \leq J, I \neq J\right\rangle \bigcap\left\langle\varepsilon_{\infty}(J)\right\rangle}\right) .
$$

From the surjections (Sur) we see that $r(J) \geq t(J)$. On the other hand, summing ranks in all the sequences $(\mathrm{ES}(\mathrm{J}))$, we recover that $\sum_{J} t(J)=\operatorname{rank}_{\Lambda_{L}}\left(\bar{C}_{\infty}\right)=$ $d(\mathcal{P})=\sum_{J} r(J)$ by 2 of lemma 6.7. Therefore all inequalities $r(J) \geq t(J)$ are actually equalities. This proves theorem 6.6.

Acknowledgements : We would like to thank A. Movahhedi for his participation to the study of case A, which was actually the starting point of this article. 
Iwasawa Descent and Co-descent for Units modulo Circular Units 495

\section{REFERENCES}

[B1] J.-R. Belliard, Sur la structure galoisienne des unités circulaires dans les $\mathbb{Z}_{p}$-extensions, J. Number Theory 69 (1998), 16-49.

[B2] J.-R. Belliard, Sous-modules d'unités en théorie d'Iwasawa, Publications Mathématiques de Besançon (2002), 12p.

[B3] J.-R. Belliard, Global units modulo circular units: descent without Iwasawa's Main Conjecture, preprint (2005).

[BN1] J.-R. Belliard, T. Nguyen Quang Do, Formules de classes pour les corps abéliens réels, Ann. Inst. Fourier, Grenoble, 51 (2001), 903-937.

[BN2] J.-R. Belliard, T. Nguyen Quang Do, On modified circular units and annihilation of real classes, Nagoya Math. J., 177 (2005), 77-115.

[C] P. Cornacchia, Fitting ideals of class groups in a $\mathbb{Z}_{p}$-extension, Acta Arithm. 87 (1998), 79-88.

[FGS] L. Federer, B.H. Gross (with an appendix by W. Sinnott), Regulators and Iwasawa modules, Invent. Math. 62 (1981), 443-457.

[FG] M. Flach (with an appendix by C. Greither), The equivariant Tamagawa Number Conjecture: A survey, Contemporary Math. 103 (1991), 9-24.

[Gb] R. Greenberg, On the Iwasawa invariants of totally real number fields, Amer. J. Math. 98 (1976), 263-284.

[Gt] C. Greither, Class groups of abelian fields, and the Main Conjecture, Ann. Inst. Fourier 42, 3 (1992), 449-499.

[Iw1] K. Iwasawa, On cohomology groups of units for $\mathbb{Z}_{p}$-extensions, Amer. J. Math. 105 (1985), 189-200.

[Iw2] K. Iwasawa, On $\mathbb{Z}_{l}$-extensions of algebraic number fields, Ann. of Math. 98 (1973), 246326.

[J] J.-F. Jaulent, Sur les conjectures de Leopoldt et de Gross, Astérisque 147-148 (1987), 107-120.

[Ko] M. Kolster, An idelic approach to the wild kernel, Invent. Math. 103 (1991), 9-24.

[KS] J. Kraft, R. Schoof, Computing Iwasawa modules of real quadratic number fields, Compositio Math. 97 no. 1-2 (1995), 135-155.

[KN] R. Kucera, J. Nekovar, Cyclotomic units in $\mathbb{Z}_{p}$-extensions, J. Algebra 171 (1995), 457472 .

[Ku] L.V. Kuz'min, The Tate module for algebraic number fields, Math. USSR Izv. 6-2 (1972), 263-321.

[LF] M. Le Floc'h, On Fitting ideals of certain étale K-groups, K-theory 27 (2002), 281-292.

[LMN] M. Le Floc'h, A. Movahhedi, T. Nguyen Quang Do, On capitulation cokernels in Iwasawa theory, Amer. J. of Math. 127 (2005), 851-877

[N] T. Nguyen Quang Do, Sur la conjecture faible de Greenberg dans le cas abélien pdécomposé, to appear in Int. J. Number Theory.

[O.I] M. Ozaki, On the cyclotomic Unit Group and the Ideal Class Group of a Real Abelian Number Field, J. Number Theory 64 (1997), 211-222.

[O.II] M. Ozaki, On the cyclotomic Unit Group and the Ideal Class Group of a Real Abelian Number Field, II, J. Number Theory 64 (1997), 223-232.

[S] W. Sinnott, On the Stickelberger Ideal and the Circular Units of an Abelian Field, Invent. Math. 62 (1981), 181-234.

[T] T. Tsuji, Semi-local units modulo cyclotomic units, J. Number Theory 78 (1999), 1-26.

[W] L. C. Washington, Introduction to Cyclotomic Fields, 2nd ed., Springer-Verlag, New York, 1997. 
Jean-Robert Belliard

Université de Franche-Comté, UMR 6623, 16, route de Gray 25030 Besançon cedex. FRANCE

E-mail: belliard@math.univ-fcomte.fr

Mikaël Lescop

Université de Limoges, LACO (UMR CNRS 6090 ), 123

Avenue Albert Thomas, 87060 Limoges cedex. FRANCE

E-mail: mikael.lescop@unilim.fr

Thong Nguyê̂n-Quang-Dô̂

Université de Franche-Comté, UMR 6623, 16

route de Gray, 25030 Besançon cedex. FRANCE

E-mail: nguyen@math.univ-fcomte.fr

fax : 0381666623 